PREPARED FOR THE U.S. DEPARTMENT OF ENERGY, UNDER CONTRACT DE-AC02-76CH03073

PPPL-3882

PPPL-3882

UC-70

Regular and Stochastic Orbits of Ions in a Highly Prolate Field-reversed Configuration

by

A.S. Landsman, S.A. Cohen, and A.H. Glasser

October 2003

$\left.\stackrel{M}{M}\right|_{\substack{\text { PRInCETON PLASIMA } \\ \text { PHYSICS LABORATORY }}} ^{D}$

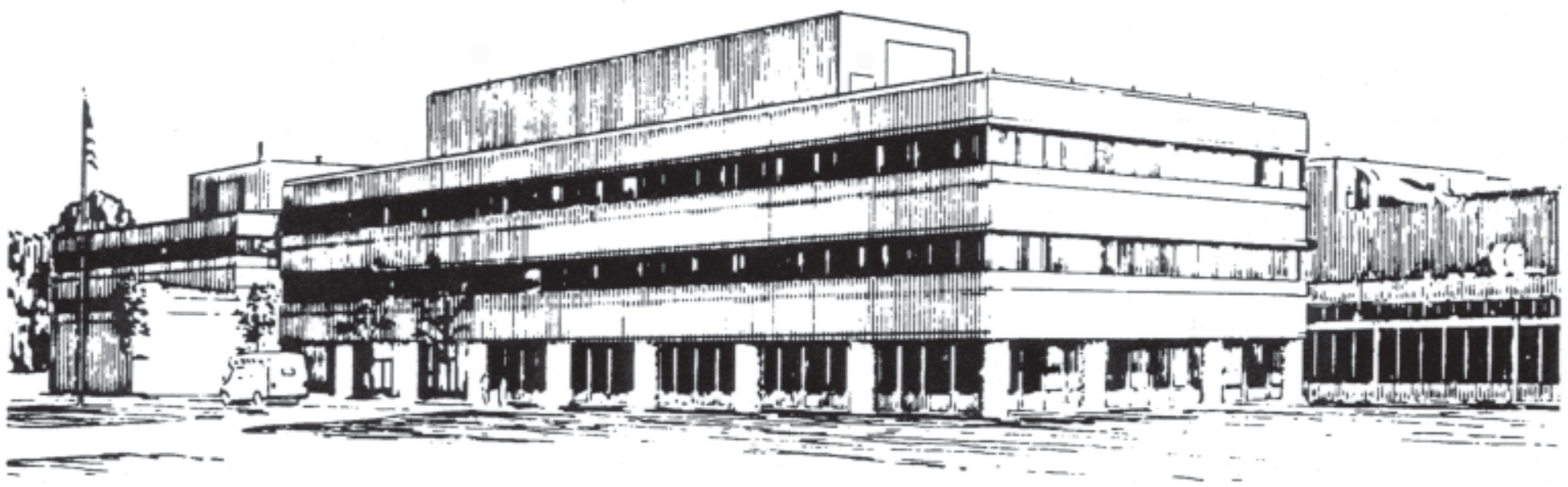

PRINCETON PLASMA PHYSICS LABORATORY PRINCETON UNIVERSITY, PRINCETON, NEW JERSEY 


\section{PPPL Reports Disclaimer}

This report was prepared as an account of work sponsored by an agency of the United States Government. Neither the United States Government nor any agency thereof, nor any of their employees, makes any warranty, express or implied, or assumes any legal liability or responsibility for the accuracy, completeness, or usefulness of any information, apparatus, product, or process disclosed, or represents that its use would not infringe privately owned rights. Reference herein to any specific commercial product, process, or service by trade name, trademark, manufacturer, or otherwise, does not necessarily constitute or imply its endorsement, recommendation, or favoring by the United States Government or any agency thereof. The views and opinions of authors expressed herein do not necessarily state or reflect those of the United States Government or any agency thereof.

\section{Availability}

This report is posted on the U.S. Department of Energy's Princeton Plasma Physics Laboratory Publications and Reports web site in Fiscal Year 2004. The home page for PPPL Reports and Publications is: http://www.pppl.gov/pub_report/

DOE and DOE Contractors can obtain copies of this report from:

U.S. Department of Energy

Office of Scientific and Technical Information

DOE Technical Information Services (DTIS)

P.O. Box 62

Oak Ridge, TN 37831

Telephone: (865) 576-8401

Fax: (865) 576-5728

Email: reports@adonis.osti.gov

This report is available to the general public from:

National Technical Information Service

U.S. Department of Commerce

5285 Port Royal Road

Springfield, VA 22161

Telephone: $1-800-553-6847$ or

(703) $605-6000$

Fax: (703) 321-8547

Internet: http://www.ntis.gov/ordering.htm 


\title{
Regular and stochastic orbits of ions in a highly prolate field-reversed configuration
}

\author{
A.S. Landsman* and S. A. Cohen \\ Princeton Plasma Physics Laboratory, \\ P.O. Box 451, Princeton, NJ 08543 \\ A. H. Glasser \\ Mail Stop K717, Los Alamos National Laboratory, \\ P.O. Box 1663, Los Alamos, NM 87545
}

(Dated: September 30, 2003)

\begin{abstract}
Ion dynamics in a field-reversed configuration (FRC) are explored for a highly elongated device, with emphasis placed on ions having positive canonical angular momentum. Due to angular invariance, the equations of motion are that of a two degree of freedom system with spatial variables $\rho$ and $\zeta$. As a result of separation of time scales of motion, caused by large elongation, there is a conserved adiabatic invariant, $J_{\rho}$, which breaks down during the crossing of the phase-space separatrix. For integrable motion, which conserves $J_{\rho}$, an approximate one-dimensional effective potential was obtained by averaging over the fast radial motion. This averaged potential has the shape of either a double or single symmetric well centered about $\zeta=0$. The condition for the approach to the separatrix and therefore the break-down of the adiabatic invariance of $J_{\rho}$ is derived and studied under variation of $J_{\rho}$ and conserved angular momentum, $\pi_{\phi}$. Since repeated violation of $J_{\rho}$ results in chaotic motion, this condition can be used to predict whether an ion (or distribution of ions) with given initial conditions will undergo chaotic motion.
\end{abstract}

PACS numbers: 52.65.Cc, 05.45.Ac, 05.45.Pq, 52.20.Dq, 52.55.Lf 


\section{INTRODUCTION}

Field-reversed configurations (FRCs) occur in plasmas of astrophysical, planetary, ${ }^{1}$ and laboratory scales. In all these cases, conditions may exist where the particles are collisionless and their Larmor orbits of size comparable to the spatial scale of the magnetic field. FiniteLarmor-radius (FLR) conditions are particularly important to the FRC as a fusion reactor, ${ }^{2}$ our primary interest. This paper elucidates the phase-space structure of particle motion in an axisymmetric prolate FRC and derives the boundary between regular and stochastic behavior for trapped-particle orbits. Understanding stochastic behavior of particle orbits in the FRC, essential to issues of macroscopic stability, ${ }^{3}$ confinement, and heating, is necessary to explain experimental observations of laboratory FRCs, ${ }^{4-7}$ such as those which show stability for times much longer than predicted by MHD theory. ${ }^{8}$

Particle orbits in FRC and FRC-like geometries have been previously studied. The phasespace structure in the $z=0$ subspace is investigated by Wang and Miley (WM) . Throughout this paper we use a cylindrical coordinate system $(r, z, \phi)$, with $r$ the radius of the device, and $z$ the distance along the axis from the midplane at $z=0$. Motion in this subspace is integrable, hence the WM study did not elucidate conditions for stochastic behavior or stability to perturbation out of the subspace. Finn, ${ }^{10}$ R.V. Lovelace, D.A. Larrabee and Fleischmann (LLF), ${ }^{3}$ and Finn and Sudan (FS) ${ }^{11}$ treat orbit stochasticity in toroidal FRCs by using perturbation techniques in situations where resonances occur between the $r$ and $z$ degrees-of-freedom. The LLF work considers oblate devices and axis-encircling orbits only. Rather than restricting attention to individual resonances and/or large gyroradius orbits, the present paper aims to find a more general condition on chaotic motion by considering the slowing-down in orbital radial frequency, $\omega_{r}$, which is shown to occur for a class of trapped particles. Boundaries between regular and chaotic motion and the structure of phase space have been explored in depth by Kim and Cary $(\mathrm{KC})^{12}$ for a linear FRC geometry. This linear geometry possesses an additional symmetry that allows "scaling away" the conserved canonical momentum in the linear direction, reducing the number of free parameters to one, ellipticity. The present paper studies ion orbits in an elongated Hill's vortex FRC where the toroidal geometry introduces an additional parameter $p_{\phi}$, qualitatively changing the ion dynamics in the device. Also, the elongated Hill's vortex is an elliptic geometry which allows exploration of the effects on orbit stability of axial field curvature, expected to provide more 
macroscopic stability than the racetrack geometry. ${ }^{13}$ The boundary for chaotic behavior in an elongated Hill's vortex is obtained computationally by Yumi, Toshiki and Yoshiomi (YTY) ${ }^{14}$ for several values of energy. An approximate analytic criteria for stochasticity applicable for many cases of interest was obtained for ions passing through the midplane by Belova, Davidson, Ji and Yamada (BDJY). ${ }^{13}$ After deriving the averaged shape of the potential, the present paper proceeds to obtain a general analytic expression for the transition to chaotic motion that can be applied to any set of initial conditions or distribution functions.

A Newtonian formulation is applied to charged particles in an elongated Hill's vortex FRC by Hugrass and Turley ${ }^{15}$ and used to study a limited range of regular orbits for the axisymmetric situation. Phase-space structure is not studied. The present paper uses a Hamiltonian approach, analytic techniques from non-linear dynamics, and more extensive single-particle numerical simulations to find the shape of the averaged potential and find a criteria for the existence of an adiabatic invariant for those orbits which do not cross the phase-space separatrix. It should not to be confused with the Hill's vortex magnetic-field separatrix, $S_{m}$, which separates open from closed field lines in configuration space.

Our numerical simulations ${ }^{16,17}$ use Hamilton's equations and follow the full threedimensional motion of a single ion. Unlike that earlier work, this paper does not treat rotating magnetic fields, leaving the system axisymmetric and time-independent. An ion moving in such fields possesses two exact invariants: energy $E$ and canonical angular momentum $p_{\phi}$. Due to a second invariant, the Hamiltonian can be reduced to four canonical variables, $r, p_{r}, z$, and $p_{z}$, and the motion can be viewed as that of a particle moving in a two-dimensional potential well, $V_{\text {eff }}(r, z)$. Since this is a two-dimensional system, the motion is generally non-integrable, except in cases where there is a third, perhaps adiabatic, invariant. A particle in a highly-elongated FRC possess an additional invariant which arises when its frequency of motion along $r$ is much higher than along $z$, leading to an adiabatic invariant, the radial action $J_{r} \equiv \oint p_{r} d r / 2 \pi$, for that class of orbits. Break-down of this invariance occurs at the crossing of the phase space separatrix ${ }^{18,19}$ that bounds cyclotron orbits from figure- 8 orbits, and which in the paper will be referred to simply as the separatrix.

Section II presents the equations of motion, a simple physical picture explaining orbit stability, and, following WM, reviews types of orbits in the FRC midplane. New types of axis-encircling orbit are classified. Section III discusses the stability of all orbit classes to small axial dispacements. By averaging over the fast motion in $r$, an approximate averaged 
one-dimensional potential is derived for regular motion. The shape of of this averaged one dimensional potential is either a double or single potential well centered about $z=0$ and depends on energy along the $r$ degree-of-freedom, $H_{r}$, and an exact invariant, $p_{\phi}$. The averaged one-dimensional potential gives a qualitative picture of ion dynamics and clarifies the trapping of particles in one of the two potential wells along $z$. Section IV uses the shape of the averaged potential to derive a general criterion for the existence of an adiabatic invariant, which leads to regular orbits. Though all the results herein could apply equally well to electrons, their orbits in most FRCs are predominantly of the small-gyroradius type which we treat only in passing. Section V summarizes our conclusions and discusses the relationship between our criterion and that of BDJY. ${ }^{13}$

\section{TYPES OF MIDPLANE PARTICLE ORBITS}

In this section we present the basic equations for the FRC field and Hamilton's equations for the particle motion, introduce appropriate dimensionless variables, and describe a classification of orbits in the FRC midplane.

For this study we use an analytic FRC equilibrium (elongated Hill's vortex or Solov'ev equilibrium $\left.^{20}\right)$ described in cylindrical coordinates $(r, z, \phi)$ by a flux function

$$
\psi(r, z)=r A_{\phi}=\psi_{0}\left(\frac{r^{2}}{r_{s}^{2}}\right)\left(1-\frac{r^{2}}{r_{s}^{2}}-\frac{z^{2}}{z_{s}^{2}}\right),
$$

with the magnetic field given by $\mathbf{B}=\nabla \times \mathbf{A}=\nabla \psi \times \nabla \phi$, the constant $\psi_{0}=B_{0} r_{s}^{2} / 2$, and $B_{0}$ the peak magnetic field strength at $r=z=0$. There is an elliptic separatrix, $S_{m}$, with radius $r_{s}$ and half-length $z_{s}$, x-points at $r=0, z= \pm z_{s}$, and an o-point at $r=r_{s} / \sqrt{2}$. $\psi$ is

positive inside $S_{m}$ and negative outside. Much of our work is based on the assumption of a highly prolate (or large elongation) FRC, where

$$
\epsilon \equiv \frac{r_{s}}{z_{s}} \ll 1
$$

The Hamiltonian for a single ion of mass $m$ and charge $q$ is given in CGS units by

$$
H\left(r, p_{r}, z, p_{z}, p_{\phi}\right)=\frac{1}{2 m}\left[p_{r}^{2}+p_{z}^{2}+\frac{1}{r^{2}}\left(p_{\phi}-\frac{q}{c} \psi\right)^{2}\right]
$$

with canonical momenta $p_{i}$, satisfying Hamilton's equations,

$$
\frac{d x_{i}}{d t}=\frac{\partial H}{\partial p_{i}}, \quad \frac{d p_{i}}{d t}=-\frac{\partial H}{\partial x_{i}}, \quad x_{i}=(r, z, \phi), \quad p_{i}=\left(p_{r}, p_{z}, p_{\phi}\right) .
$$


Since $H$ is independent of $\phi, p_{\phi}$ is conserved, and the system describes motion with two degrees-of-freedom in an positive-definite effective potential

$$
V_{\mathrm{eff}} \equiv \frac{1}{2 m r^{2}}\left(p_{\phi}-\frac{q}{c} \psi\right)^{2}
$$

To simplify the discussion and elucidate the physics, we define scale factors,

$$
\Omega_{0} \equiv \frac{q B_{0}}{m c}, \quad V_{0} \equiv \frac{m \Omega_{0}^{2} r_{s}^{2}}{8}, \quad p_{0} \equiv\left(2 m V_{0}\right)^{1 / 2}
$$

$\Omega_{0}$ is the cyclotron frequency of a particle in a uniform magnetic field of strength $B_{0} ; V_{0}$ is the energy of a particle in such a field with a gyration radius of $r_{s} / 2$; and $p_{0}$ is the momentum of a particle with kinetic energy $V_{0}$. Next we define scaled displacements and canonical momenta,

$$
\rho \equiv \frac{r}{r_{s}}, \quad \zeta \equiv \frac{z}{z_{s}}, \quad \pi_{\rho} \equiv \frac{p_{r}}{p_{0}}, \quad \pi_{\zeta} \equiv \frac{p_{z}}{p_{0}}, \quad \pi_{\phi} \equiv \frac{p_{\phi} c}{q \psi_{0}}
$$

$\rho$ and $\zeta$ are the fractional radial and axial distances to the magnetic separatrix. With these definitions, the effective potential can be expressed as

$$
V_{\text {eff }}=V_{0} v\left(\rho, \zeta, \pi_{\phi}\right), \quad v\left(\rho, \zeta, \pi_{\phi}\right) \equiv \frac{f^{2}\left(\rho, \zeta, \pi_{\phi}\right)}{\rho^{2}}, \quad f\left(\rho, \zeta, \pi_{\phi}\right) \equiv \pi_{\phi}-\rho^{2}\left(1-\rho^{2}-\zeta^{2}\right)
$$

and the Hamiltonian becomes

$$
H=V_{0} \varepsilon, \quad \varepsilon \equiv \pi_{\rho}^{2}+\pi_{\zeta}^{2}+v
$$

Applying Hamilton's equations to Eq. (3), we obtain

$$
\dot{\phi}=\frac{\Omega_{0}}{2} \frac{f\left(\rho, \zeta, \pi_{\phi}\right)}{\rho^{2}}
$$

Thus $\dot{\phi}$ changes sign when $f$ (and therefore $v$ ) vanishes. Applying Hamilton's equations, Equation (4), to the Hamiltonian, Equation (9), where the partial derivative of $H$ is taken with respect to $\zeta$, we get a force along $\zeta$ :

$$
\ddot{\zeta}+\omega_{\zeta}^{2} \zeta=0, \quad \omega_{\zeta}^{2} \equiv \epsilon^{2} \Omega_{0} \rho^{2} \dot{\phi}=\epsilon^{2} \frac{\Omega_{0}^{2}}{2} f\left(\rho, \zeta, \pi_{\phi}\right)
$$

From the equation above, it can be seen that at $\zeta=0$, the ion does not experience a force along $\zeta$, so that given the initial condition $\zeta=\pi_{\zeta}=0$, the ion will remain in the $\zeta=0$ subspace. Equation (11) has a simple harmonic form, though with a non-constant coefficient 
multiplying $\zeta$. For low-energy cyclotron orbits, the frequency $\omega_{\rho}$ is close to the cyclotron frequncy, $\Omega_{0}$. Unlike $\omega_{\zeta}$ in Eq. (11), $\omega_{\rho}$ is not proportional to $\epsilon$, so that for small values of $\epsilon$, there is a separation of frequencies with $\omega_{\rho} \gg \omega_{\zeta}$. This holds except at the approach to separatrix, where $\omega_{\rho}$ slows down. This case and the resultant violation of an adiabatic invariant will be discussed later in this paper. For orbits not close to the separatrix, $\omega_{\rho} \gg \omega_{\zeta}$ holds, so that we can average $\rho^{2} \dot{\phi}$ (see Eq. (11)) over one period of oscillation in $\rho$ to obtain an averaged force along $\zeta$. In general, the fast motion can be averaged whenever there is a large seperation in frequencies of motion. ${ }^{22}$ Since $\omega_{\zeta}^{2} \propto \rho^{2} \dot{\phi}$, the $\zeta$-motion is stable or unstable to perturbations from $\zeta=0$, depending on the sign of $\left\langle\rho^{2} \dot{\phi}\right\rangle$.

Consider orbits in the invariant subspace $\zeta=\pi_{\zeta}=0$, some of which have been explored by WM. ${ }^{9}$ The orbits are that of a particle in a 1-D effective potential $V_{\text {eff }}=V_{0} v\left(\rho, 0, \pi_{\phi}\right)$ and are therefore integrable. Figure 1 shows four possible shapes of the scaled potential energy $v$ for representative values of $\pi_{\phi}$. Figure 2 shows particle orbits projected onto the $\rho$ - $\phi$ plane for each of these cases. Our orbit classification is presented in order of increasing values of $\pi_{\phi}$

- Case I. $\pi_{\phi}<-1 / 12$. The scaled potential energy $v\left(\rho, 0, \pi_{\phi}\right)$ has a minimum $v\left(\rho, 0, \pi_{\phi}\right)=0$ at $\rho^{2}=1 / 2+\left(1 / 4-\pi_{\phi}\right)^{1 / 2}$ and $\rightarrow \infty$ for $\rho \rightarrow 0, \infty$, resulting in a single well. Figure 2-I shows three representative orbits for this case, with low, medium, and high energy. Orbits (a), with $\varepsilon=0.01$, and (b), with $\varepsilon=0.2$, are both cyclotron orbits outside the o-point at $r=7.07$. They have negative average $\rho^{2} \dot{\phi}$ and are therefore unstable to small perturbations in $\zeta$. Orbit (c), with $\varepsilon=0.4$, extends over a wider range of $\rho$, including both inside and outside the o-point, forming a figure- 8 . The orbit shown has negative $<\rho^{2} \dot{\phi}>$, but similar shaped orbits may have positive $<\rho^{2} \dot{\phi}>$.

- Case II. $-1 / 12<\pi_{\phi}<0$. In addition to the zero of $v$ as in case I, there are extrema at $\rho^{2}=\left[1 \pm\left(1+12 \pi_{\phi}\right)^{1 / 2}\right] / 6$, resulting in a double well. Figure 2-II shows 3 representative orbits for this case. Orbit (a), with $\varepsilon=0.1$, is trapped near the bottom of the outer well. Orbit (b), with $\varepsilon=0.18$, oscillates about the inner minimum and is axis-encircling orbit. Orbit (c), with $\varepsilon=0.4$, extends over both minima, forming a figure- 8 with the inner lobe encircling the axis. All of these orbits have negative $<\rho^{2} \dot{\phi}>$ and are therefore unstable to small perturbations in $\zeta$. 
- Case III. $0<\pi_{\phi}<1 / 4$. There are two minima where $v=0$, at $\rho^{2}=1 / 2 \pm(1 / 4-$ $\left.\pi_{\phi}\right)^{1 / 2}$. Figure 2-III shows 3 representative orbits for this case. Orbits (a) and (b) both have the same scaled energy $\varepsilon=0.05$, with (a) oscillating about the outer well, outside the o-point, and (b) about the inner well, inside the o-point. Both execute cyclotron orbits, and both have negative $<\rho^{2} \dot{\phi}>$. Orbit (c), with $\varepsilon=0.2$, extends over both wells, resulting in a figure- 8 shape, with positive average $\rho^{2} \dot{\phi}$. This is the case discussed in great detail in this paper, since the transition between cyclotron and figure- 8 orbits which occurs in this range of $\pi_{\phi}$ and the resultant crossing of the separatrix results in the break-down of an adiabatic invariant which is otherwise conserved in this low $\epsilon$ system. Thus, from now on, positive $\pi_{\phi}$ figure- 8 orbits will be referred to simply as figure-8 orbits.

- Case IV. $1 / 4<\pi_{\phi}$. There is a single potential well with a raised minimumum, $v>0$. Figure 2-IV shows 3 representative orbits for this case, with $\varepsilon=0.035,0.2$, and 0.4 . All betatron orbits have $\dot{\phi}>0$.

\section{ORBIT STABILITY AND THE $r$-AVERAGED POTENTIAL}

Transitions between the orbit types enumerated in Section II may occur when particles move out of the $\zeta=\pi_{\zeta}=0$ subspace. Accordingly, we now consider the stability of orbits to perturbations out of the this subspace.

Figure 3 shows the shape of $v\left(\rho, \zeta, \pi_{\phi}\right)$ vs. $\rho$ for four elevations in $\zeta$ for Case III. The radial zeroes of $v$ are found from Eq. (8),

$$
\rho^{2}=\frac{1}{2}\left\{\left(1-\zeta^{2}\right)^{2} \pm\left[\left(1-\zeta^{2}\right)^{2}-4 \pi_{\phi}\right]^{1 / 2}\right\}
$$

$\dot{\phi}$ is negative in the interval between the two zeroes and positive elsewhere. For the raised potential it is everywhere positive. From Eq. (11), the force along $\zeta$ is stabilizing for $\dot{\phi}>0$, thus the raised potential well exerts a stabilizing force on the ion. Eq. (12) shows that the destabilizing region between the zeroes vanishes for $\zeta^{2}>1-\left(4 \pi_{\phi}\right)^{1 / 2}$. It follows that any ion with $\pi_{\phi}>0$ moving to larger $|\zeta|$ is subject to a restoring force towards the $\zeta=0$ midplane as the central barrier in the double potential well lowers, as seen in Fig. 3, reducing the time spent by the ion in the negative $\dot{\phi}$ part of the trajectory. With a finite $\zeta$-velocity directed away from the midplane, the positive $\pi_{\phi}$ ion eventually will reach a region of $\zeta$ where $v(\rho, \zeta)$ 
has the shape of a raised single potential well. There $\dot{\phi}$ will be positive everywhere along the trajectory and ion will experience a positive restoring force at all points along its trajectory. In ion dynamics, the traversal over the barrier between wells and the resultant crossing of the separatrix corresponds to a change of orbit from cyclotron to figure- 8 . If the initial $\pi_{\zeta}$ is high enough for the particle to reach the single raised-potential-well region, the orbit will then become betatron. The restoring force along $\zeta$, proportional to the averaged $\rho^{2} \dot{\phi}$, increases with absolute value of $\zeta$.

For negative $\pi_{\phi}$ orbits (cases I and II, Figure 1 I,II), $\dot{\phi}<0$ on the left part of the trajectory along $\rho$, since $v\left(\rho, \zeta, \pi_{\phi}\right)$ has only one zero in this range of $\pi_{\phi}$, so that $\dot{\phi}$ changes sign only once. In this case, ion motion to higher absolute value of $\zeta$ does not necessarily increase the restoring force, as occurs in the $\pi_{\phi}>0$ cases, III and IV.

For a highly prolate (small $\epsilon$ ) FRC, $\omega_{\rho} \gg \omega_{\zeta}$ for all orbits whose energy is not too close to the separatrix or the top of the potential barrier that divides the double well. In other words, the condition $\omega_{\rho} \gg \omega_{\zeta}$ applies for all orbits which are not close to the transition between cyclotron and figure-8 motion, since such a transition coincides with a slow-down of frequency $\omega_{\rho}$, much as the frequency of a pendulum slows down as it approaches the top along a trajectory that separates oscillating from circulating motion. In cases where the condition $\omega_{\rho} \gg \omega_{\zeta}$ holds, the average force constant for motion along $\zeta$ can be found by using Eq. (11) and integrating $\epsilon^{2} \Omega_{0} \int \rho^{2} \dot{\phi} d t$ over one period of oscillation in $\rho$. This provides a qualitative picture of the potential well in which an ion moves after averaging over fast motion in $\rho$. Since the sign of the radial-weighted average azimuthal drift, $\left\langle\rho^{2} \dot{\phi}>\right.$ determines the stability to perturbation out of the $\zeta=0$ plane, cyclotron orbits which do not encircle the major axis, i.e., type $a$ and $e$, all of which have a clockwise angular drift, are unstable. High energy figure- 8 orbits drift counter-clockwise and are therefore stable to perturbations; lower energy figure- 8 orbits drift clockwise and are therefore unstable to perturbations out of the subspace. The clockwise drift is due to the fact that lower energy figure- 8 orbits spend more of their time near the central barrier separating the double potential (see Case III in Figure 1 where $\dot{\phi}$ is negative and therefore get a net negative angular drift. The fact that the central barrier in the symmetric double potential gives a negative contribution to angular drift also explains why positive $\pi_{\phi}$ ions show better confinement. The symmetric double well becomes more narrow and the potential barrier drops with increasing absolute value of $\zeta$, as shown in Figure 3. As cyclotron orbits move away from $\zeta=0$, their energy 
is eventually high enough to cross the separatrix whose area shrinks with increasing $|\zeta|$ and turn into figure- 8 orbits. As the barrier along $\rho$ falls further, they will eventually turn into figure- 8 orbits with positive average $\rho^{2} \dot{\phi}$, feeling an attractive force towards $\zeta=0$.

For orbits away from the separatrix, where $\omega_{\rho} \gg \omega_{\zeta}$, we can obtain a quantitative picture of the averaged potential along $\zeta$ in which an ion moves after averaging over the fast motion in $\rho$. Segregating the $\zeta$-dependent parts to Eq. (7) yields:

$$
v\left(\rho, \zeta, \pi_{\phi}\right)=-\left[\rho^{2}\left(1-\rho^{2}\right)-\pi_{\phi}\right] \zeta^{2}+\frac{\rho^{2}}{2} \zeta^{4}+g(\rho)
$$

where $v\left(\rho, \zeta, \pi_{\phi}\right)$ is a scaled potential (Eq. 8) Where the $\zeta$ independent parts of the expression are segregated under $g(\rho)$ For regular motion, a new effective scaled potential, $\langle v(\zeta)>$, is obtained after averaging over fast oscillations in $\rho$. The variable of fast motion, $\rho$, drops out and $\pi_{\phi}$ is always a constant of motion, so that $<v(\zeta)>$ represents averaged motion in a one-dimensional potential along $\zeta$. The shape of the averaged potential along $\zeta$ for $\pi_{\phi}>0$ ions is either a single or a double well centered around $\zeta=0$. Figure $4 \mathrm{a}$ ) and b) show numerically calculated phase-space plots, $p_{0} \pi_{\zeta} v s . z_{s} \zeta$ for higher and lower amplitudes of oscillation along $\rho$, respectively. It can be seen that the potential along $\zeta$ is either a single or a symmetric double potential well, with the location of the minima determined by $\pi_{\phi}$ and the energy of oscillation along $\rho$. Fast oscillations due to the fast $\rho$ motion are superimposed on the closed curves in $\pi_{\zeta}$ vs. $\zeta$ phase space in Figure $4 . J_{\rho}$ is a scaled adiabatic invariant, which will be discussed in much detail in the next section, and the motion is regular.

A figure-8 orbit can be approximated as executing a non-linear $\zeta$-dependent oscillation:

$$
\rho=\rho_{h}+\sum_{n=1}^{\infty} A_{n} \cos n \omega\left(t-t_{0}\right),
$$

where $\rho_{h}$ is the location of the top of potential barrier that separates the two potential wells for $0<\pi_{\phi}<\frac{1}{4}$, see Figure 1 . It is computed by finding $\partial v\left(\rho, \zeta, \pi_{\phi}\right) / \partial \rho=0$ where $\partial^{2} v\left(\rho, \zeta, \pi_{\phi}\right) / \partial \rho^{2}<0$ :

$$
\rho_{h}^{2}=\frac{1}{6}\left\{\left(1-\zeta^{2}\right)+\left[\left(1-\zeta^{2}\right)^{2}+12 \pi_{\phi}\right]^{1 / 2}\right\}
$$

Keeping only the lowest order terms in Eq. (10) and dropping the subscript on $A_{n}$ :

$$
\rho \approx \rho_{h}+A \cos \omega\left(t-t_{0}\right)
$$


where $\rho_{h}, A$, and $\omega$ are functions of $\zeta$. Calculating averaged terms, $<\rho^{2}>=\rho_{h}^{2}+\frac{1}{2} A^{2}$ and $<\rho^{4}>=\rho_{h}^{4}+\frac{5}{2} \rho_{h}^{2} A^{2}+\frac{3}{8} A^{4}$, and substituting for $\rho$ into Eq. (13) produces, after dropping all $\zeta$-independent and oscillatory terms,

$$
<v(\zeta)>\approx \zeta^{4}\left(\frac{\rho_{h}^{2}}{2}+\frac{A^{2}}{4}\right)-\zeta^{2}\left(\rho_{h}^{2}\left(1-\rho_{h}^{2}\right)-\pi_{\phi}+\frac{A^{2}}{2}\left(1-6 \rho_{h}^{2}\right)-\frac{3}{8} A^{4}\right)
$$

The first term in the coefficient for $\zeta^{2}$ is positive since $\rho_{h}<1$ and it can be shown graphically, Figure 5 , that at $\zeta=0$

$$
\rho_{h}^{2}\left(1-\rho_{h}^{2}\right)-\pi_{\phi}>0
$$

for all $0<\pi_{\phi}<\frac{1}{4}$.

Using Eq. (15), it can be easily shown that for $2 \zeta^{2}<1$

$$
1-6 \rho_{h}^{2}<0
$$

at all value of $\pi_{\phi}$. It follows that the contributions from amplitude of oscillation terms, $A$, to $\zeta^{2}$ are positive, at least for smaller values of $\zeta$. Thus for higher amplitudes of oscillation along $\rho$ such that

$$
\rho_{h}^{2}\left(1-\rho_{h}^{2}\right)-\pi_{\phi}<-\frac{A^{2}}{2}\left(1-6 \rho_{h}^{2}\right)+\frac{3}{8} A^{4}
$$

the coefficient multiplying $\zeta^{2}$ is positive and $\zeta=0$ is a global stable minima of the $\rho$-averaged motion. Thus at higher amplitudes of oscillation, $A$, along $\rho$, the averaged motion along $\zeta$ is in a single well with a potential given by $\left\langle v(\zeta)>\right.$. At lower values of $A$, the $\zeta^{2}$ term in Eq. (17) gives a negative contribution, resulting in a double well, symmetric about $\zeta=0$. To get an approximation for the location of the minima of this averaged symmetric double well, we can approximate $A$ and $\rho_{h}$ as constants. For $\zeta^{2} \ll 1$, Eq. (15) can be approximated as:

$$
\rho_{h}^{2} \approx\left(K_{1}-K_{2} \zeta^{2}\right)
$$

where $K_{1}=\frac{1}{6}\left(1+C_{1}\right), K_{2}=\frac{1}{6}\left(1+\frac{1}{C_{1}}\right)$, and $C_{1}=\left(1+12 \pi_{\phi}\right)^{\frac{1}{2}}$. It follows that $K_{1}>K_{2}$ for all positive $\pi_{\phi}$. Dropping the second term:

$$
\rho_{h}^{2} \approx K_{1}
$$

The criterion $\zeta^{2} \ll 1$ is a reasonable assumption, except in cases where $A$ is low and $\pi_{\phi}<\frac{1}{8}$. This is due to the fact that lower values of $A$ and $\pi_{\phi}$ mean higher $|\zeta|$ for the location of 
a minima. Substituting Eq. (22) into Eq. (17) and assuming $A$ is independent of $\zeta$, we see that for smaller $A$ the coefficient of $\zeta^{2}$ in Eq. (17) is negative, and $\langle v(\zeta)\rangle$ can be approximated as

$$
<v(\zeta)>=D \zeta^{4}-L \zeta^{2}
$$

where $D$ and $L$ are positive coefficients. Completing the square shows that the potential along $\zeta$ has minima at $\zeta= \pm \sqrt{\frac{L}{2 D}}$, resulting in a symmetric double potential well along $\zeta$.

For $\pi_{\phi}>\frac{1}{4}$, the coefficient $L$ is negative, even for $A=0$, thus $\langle v(\zeta)\rangle$ is minimized at $\zeta=0$ and all betatron orbits oscillate in a single potential well in $\zeta$. This is perhaps not surprising since $\pi_{\phi}>\frac{1}{4}$ is a raised potential in $\rho$, so $\rho^{2} \dot{\phi}>0$ everywhere along the orbit, exerting a stabilizing force, Eq. (11).

It can be seen from Eq. (17) that for higher values of $\pi_{\phi}, L$ in Eq. (23) is smaller and the two minima in $\langle v(\zeta)>$ are located closer together. This can be explained by the fact that the potential barrier that separates the two minima of the double potential in $\rho$ is lowered with increasing $\pi_{\phi}$, thus the time spend in the destabilizing region, where $\dot{\phi}<0$, is lessened, leading to stabilization at lower $|\zeta|$.

Figure 6 shows the ion kinetic energy along $\zeta, V_{0} \pi_{\zeta}^{2}$, vs. $\zeta$, from numerical simulation using the full Hamiltonian equations and also from motion in the approximate averaged potential given by Eq. (17) with $\rho_{h}$ given by Eq. (21). Agreement between the two is good at smaller absolute values of $\zeta$ where the $\zeta^{2} \ll 1$ assumption holds. The full Hamiltonian simulation shows a smaller maximum excursion along $\zeta$ than that of the estimate, Eq. (19), which doesn't take account of higher order terms in $\zeta$.

The above derivation assumed figure- 8 orbits. However, as previously noted all cyclotron orbits eventually move into the $\zeta$ region where the barrier of the double potential along $r$ is sufficiently low so that they are able to cross it and there become figure- 8 orbits. Then the same approximation as used in Eq. (17) can be applied with $\rho_{h}$ evaluated at the location of transition of cyclotron to a figure- 8 orbit. Figure 7, discussed more fully in the next section, shows the $\zeta$ location, as a function of $J_{\rho}$ where the transition of cyclotron to figure- 8 orbits occurs for different values of $\pi_{\phi}$.

For a particular orbit, the $\zeta$ location of a minima in $\langle v(\zeta)>$ occurs where the average $\rho^{2} \dot{\phi}$ over one period of oscillation in $\rho$ is equal to zero. Higher amplitudes of oscillation, $A$, along $\rho$ lead to lower absolute values of $\zeta$ at which the minima in $\langle v(\zeta)>$ occurs since the amount of time spend in a $\dot{\phi}>0$ part of the trajectory increases relative to time spend in 
the $\dot{\phi}<0$ part of a trajectory for higher values of $A$, see Figure 1 , curve III. Thus higher values of $A$ or higher values of $\pi_{\phi}$ result in a more closely spaced minima of the double potential well in $\zeta$, until the two wells merge at $\zeta=0$.

\section{SEPARATRIX CROSSING AND BREAK-DOWN OF THE ADIABATIC IN- VARIANT}

The dynamics described in the previous section for ion motion in both single or double effective-potential wells in $\zeta$ apply to cases of integrable motion. For cases where $\omega_{\rho} \gg \omega_{\zeta}$, there exists an adiabatic invariant, $J_{\rho}$, which is conserved up to an order of $\epsilon$ ( $\epsilon$ is defined in Eq. (2)). ${ }^{18,19} J_{\rho}$ is the area enclosed by a contour of constant energy, keeping $\zeta$ and $\pi_{\zeta}$ constant:

$$
J_{\rho} \equiv \frac{1}{2 \pi} \oint \pi_{\rho} d \rho
$$

$J_{\rho}$ is the scaled action:

$$
J_{\rho}=\frac{J_{r}}{p_{0} r_{s}}
$$

For a large elongation device, $J_{\rho}$ is adiabatically conserved except during the crossing of the separatrix, which results in transitions between figure- 8 and cyclotron orbits. Cyclotron orbits feel an average force away from $\zeta=0$, so that as the ion moves towards higher $|\zeta|$ values, its orbit will begin to approach the top of the barrier that separates the two wells in $\rho$, Figure 3, and its frequency $\omega_{\rho}$ will slow down. For smooth Hamiltonians, the nonlinear frequency $\omega_{\rho}$ near the separatrix has the following form ${ }^{21}$ :

$$
\omega_{\rho}(h)=\frac{F \Omega_{0}}{\ln (G /|h|)}
$$

where $F$ and $G$ are constants that depend on $V_{\text {eff }}, h \equiv \frac{\left(E-E_{s}\right)}{E_{s}}$, and $E_{s}$ is the $\rho$ directed energy at the separatrix: $E_{s}=V_{0} v\left(\rho_{h}, \zeta, \pi_{\phi}\right)$. At the approach to the separatrix, the frequency $\omega_{\rho}$ slows down. Under these conditions, the $\omega_{\rho} \gg \omega_{\zeta}$ criterion no longer holds and $J_{\rho}$ conservation is violated, resulting in stochastic motion. Since this type of transition occurs for nearly all orbits that are at any point cyclotron, it can be concluded that such orbits are, in general, non-integrable. There is a very small fraction of very low-energy cyclotron orbits where the guiding-center theory approximation applies. Excluding this small class of cyclotron orbits, a positive $p_{\phi}$ ion must, for all times, be in a figure- 8 or betatron orbit for 
integrable motion. Comparing Eqs. (11) and (26) we can see that $\omega_{\rho} \gg \omega_{\zeta}$ condition begins to break down around

$$
\epsilon \frac{\ln (G /|h|)}{F} \sim O(1)
$$

Rearanging the above equation, we can obtain an approximate form for the distance from the separatrix (in terms of dimensionless energy) at which the adiabatic invariance of $J_{\rho}$ begins to break down. Labeling this as $\delta$, where $\delta$ is just the value of $h$ below which $J_{\rho}$ is violated, we get

$$
\delta \sim O\left(G e^{\frac{-F}{\epsilon}}\right)
$$

In numerical simulations we found that $\delta \sim .05$ is sufficient to ensure the adiabatic conservation of $J_{\rho}$.

For figure- 8 orbits, $J_{\rho}$ will be conserved in one of two cases:

Case $A$ : The action $J_{\rho}$ is high enough so that the ion executes a figure- 8 orbit with $\omega_{\rho} \gg \omega_{\zeta}$ when it passes $\zeta=0$ :

$$
J_{\rho}>\frac{1}{2 \pi} \oint\left((1+\delta) v\left(\rho_{h}, 0, \pi_{\phi}\right)-\left(\frac{\pi_{\phi}}{\rho}-\rho\left(1-\rho^{2}\right)\right)^{2}\right)^{\frac{1}{2}} d \rho
$$

The action $J_{\rho}$ is evaluated in the $\zeta=0$ subspace when the energy along $\rho$ is just high enough for the ion to pass over the energy barrier at some finite speed and execute figure8 orbits. The factor $(1+\delta)$, with $\delta \sim 0.05$, ensures that the motion is not too close to the phase-space separatrix. Figure 4a) b) are examples of such orbits. A stochasticity criterion for untrapped particle ions was also derived by BDJY. ${ }^{13}$ It sets the boundary on stochastic motion by requiring the total energy to be above a certain value for a given value of $\pi_{\phi}$. The BDJY criterion while working well for most cases of interest, would fail under certain conditions due to the fact that it uses the total energy $H$, rather than the energy of $\rho$-oscillation, which determines $J_{\rho}$.

Case B: A more complex case of $J_{\rho}$ adiabatic invariance occurs at lower values of $J_{\rho}$ which do not satisfy the criterion of Eq. (21). In this case $J_{\rho}$ can still be conserved if an ion oscillates in one of the two potential wells in $\zeta$ with an amplitude low enough so that it doesn't pass into the forbidden lower region of $|\zeta|$, where the crossing of the separatrix resulting in a transition to a cyclotron orbit occurs. Figures 8 and 9 show $\pi_{\zeta} v s . \zeta$ and the corresponding Poincare plots for integrable and stochastic motion respectively, for amplitudes of $\zeta$ oscillation just below and just above the critical. 
Let us refer to the amplitude of $\zeta$ oscillation about one of its non-zero minima along $\zeta$, given by $\langle v(\zeta)>$, as the critical amplitude, if above this amplitude the ion gets close to the separatrix (where how close is determined by $\delta$, which was found numerically $\delta \sim .05$ ). This critical amplitude of $\zeta$ oscillation, below which the orbits are integrable, is a function of $J_{\rho}$. Critical values of $\zeta$ where the approach to the separatrix occurs are plotted as a function of $J_{\rho}$ in Figure 7. The actual crossing of the separatrix corresponds to a transition of a figure- 8 to cyclotron orbit. The curves were obtained by computing $J_{\rho}$ of an orbit in the vicinity of the separatrix for a set of values of $\zeta$.

$$
J_{\rho}=\frac{1}{\pi} \int_{\rho_{\min }}^{\rho_{\max }}\left\{v\left(\rho_{h}, \zeta, \pi_{\phi}\right)-\left[\frac{\pi_{\phi}}{\rho}-\rho\left(1-\rho^{2}-\zeta^{2}\right)\right]^{2}\right\}^{1 / 2} d \rho
$$

where $\zeta$ and $\rho_{h}$ are kept constant during integration $\left(\rho_{h}\right.$ is a function of $\zeta$ given by Eq. (15)). Eq. (30) was evaluated for different values of $\pi_{\phi}$ to find $J_{\rho}$ for a table of values of $\zeta$, Figure 7. The $\zeta$-intercept in Figure 7 coincides with the point where the potential barrier in $\rho$ disappears and a single raised potential forms, the condition necessary for betatron orbits which feel a force towards $\zeta=0$ in all cases. It therefore follows that, for a given value of $\pi_{\phi}$, the $\zeta$ intercept in Figure 7 is the highest possible location of a minima in $\langle v(\zeta)>$. Since it is also the location of the critical amplitude for $J_{\rho}=0$ in Figure 7, it can be seen that the critical amplitude of $\zeta$-oscillation goes to zero as $J_{\rho}$ goes to zero, resulting in a single stationary orbit for each value of $\pi_{\phi}$.

To check that the oscillations above the critical amplitude in one of the two minima along $\zeta$ result in stochastic orbits, numerical simulations using the full Hamiltonian code were made. For example, at $p_{\phi}=3 / 16$, numerical simulations of orbits at four values of $J_{\rho}$ were made, each for 5-10 closely spaced values of $\zeta$. The red stars indicate cases where the orbits were stochastic and the blue stars where the orbits were integrable. These results show the approximate conservation of the adiabatic invariant $J_{\rho}$ for oscillations in $\zeta$ below the critical amplitude.

Figures 10 and 11 show $\pi_{\zeta}$ vs. $\zeta($ Eq. $(7))$ and $J_{\rho}$ vs. $t / \tau_{c i}$ for critical amplitudes of $\zeta$ oscillation for two values of $J_{\rho}$. It can be seen that $J_{\rho}$ is approximately conserved (fluctuates by less than 10 percent). The figures show that higher values of $J_{\rho}$ have a greater range of amplitudes along $\zeta$ and approach closer to $\zeta=0$. Thus, all else being equal, particles with higher $J_{\rho}$ will have regular motion for a greater range of $\pi_{\zeta}$ and $|\zeta|$-values.

For a given value of $\pi_{\phi}$, the highest possible value of $\zeta$ where the minimum of $\langle v(\zeta)>$ 
occurs can be found by setting the square root in Eq. (12) to zero and solving for $\zeta$. This also gives the y-intercept in Figure 7:

$$
\zeta^{2}=1-2 \pi_{\phi}^{1 / 2}
$$

Using the fact that the total energy, $H$ is conserved and that $J_{\rho}$ is an adiabatic invariant, except at the crossing of the separatrix, and requiring that $\pi_{\zeta}^{2}=0$ at or below the critical amplitude (to avoid the crossing of the separatrix), we can now derive a condition for trapped particle orbits that adiabatically conserve $J_{\rho}$. This is done by finding an upper limit on energy of oscillation along $\zeta$ in the averaged potential $\langle v(\zeta)>$, for a given value of $J_{\rho}$. As was shown in the previous section, $J_{\rho}$ or identically the amplitude of oscillation along $\rho, A$, determine $\langle v(\zeta)>$. Since the total energy of an adiabatic system can be expressed as a function of $J_{\rho}, \zeta$ and $\pi_{\zeta}$, an upper limit on $H$ can be found that for a given value of $J_{\rho}$ will ensure that the energy of oscillation along $\zeta$, determined by $\zeta$ and $\pi_{\zeta}$ does not exceed critical amplitude. The condition for the adiabatic conservation of $J_{\rho}$ for trapped particle orbits thus becomes

$$
H<(1+\delta) V_{0} v\left(\rho_{h}\left(J_{\rho}\right), \zeta_{c}\left(J_{\rho}\right), \pi_{\phi}\right)
$$

where $\zeta_{c}$ can be expressed in terms of the adiabatic invariant $J_{\rho}$, and similarly $\rho_{h}$, which is evaluated at $\zeta_{c}$, can also be expressed in terms of $J_{\rho}$. The inequality above thus imposes a constraint on $H$ determined only by the constant of motion $\pi_{\phi}$ and the adiabatic invariant $J_{\rho}$. If this constraint is satisfied, $J_{\rho}$ will be a conserved adiabatic invariant of trapped ion orbits. To obtain this constraint in terms of $J_{\rho}$, we begin by estimating the location of the critical amplitude, $\zeta_{c}$, as a function of $J_{\rho}$. These values are plotted in Figure 7 for four values of $\pi_{\phi}$. From this figure we can obtain an over-estimate on $\zeta_{c}$ in all cases by approximating the curves as

$$
\zeta_{c}=C\left(J_{\max }-J_{\rho}\right)^{1 / 2}
$$

where

$$
C=\frac{\left(1-2 \pi_{\phi}{ }^{1 / 2}\right)^{1 / 2}}{\sqrt{J_{\max }}},
$$

An over-estimate on $\zeta_{c}$ is needed since it ensures that the separatrix crossing will not occur. $C$ was obtained by solving $1-2 \pi_{\phi}^{1 / 2}=C J_{\max }^{1 / 2}$ where $1-2 \pi_{\phi}^{1 / 2}$ is the $\zeta$ intercept in Figure 7 (see Eq. (31)) and $J_{\max }$ is evaluated at $\zeta=0$ for $V_{\text {eff }}=V_{0} v\left(\rho_{h}, 0, \pi_{\phi}\right)$. Substituting for $\zeta_{c}$ from Eq. (33) into Eq. (32), where $v\left(\rho_{h}, \zeta_{c}, \pi_{\phi}\right)$ is defined in Eq. (8), using $\rho_{h}^{2}$, evaluated at 
$\zeta=\zeta_{c}$, given by Eq. (21) and expanding $1 / \rho_{h}^{2}$, we get, after keeping only lowest order terms of order $\zeta_{c}^{2}$ and simplifying the expression

$$
H<(1+\delta) V_{0} \frac{1}{K_{1}}\left(A_{1}-A_{2} C^{2}\left(J_{\max }-J_{\rho}\right)\right)
$$

where

$$
\begin{gathered}
A_{1}=\left(\pi_{\phi}-K_{1}\left(1-K_{1}\right)\right)^{2} \\
A_{2}=A_{1} \frac{K_{2}}{K_{1}}+2 A_{1}{ }^{1 / 2}\left(K_{2}\left(1-K_{1}\right)+K_{1}\left(1-K_{2}\right)\right)
\end{gathered}
$$

Thus, given an ion's $J_{\rho}, \mathrm{H}$, and $\pi_{\phi}$, Eq. (35) determines whether $J_{\rho}$ is an adiabatic invariant for a trapped figure- 8 orbit. Case A, in the beginning of this section, places a limit on $J_{\rho}$ for untrapped ion orbits, above which $J_{\rho}$ is an adiabatic invariant, for those types of orbits. As previously mentioned $J_{\rho}$ invariance breaks down for all cyclotron orbits. The location along $\zeta$ of a transition of a cyclotron to a figure- 8 orbit is given by Eq. (33). At $|\zeta|$ below $\zeta_{c}$, the orbit is cyclotron, and above it is a figure-8. It is clear that the above approximation is not valid at low $\pi_{\phi}$ and energies where the $\zeta^{2} \ll 1$ assumption breaks down. For cyclotron orbits, the action $J_{\rho}$ is the same as the scaled magnetic moment $\hat{\mu}$, where $\hat{\mu}=\mu / p_{0} r_{s}$. They are abiabatic invariants up to the point of a transition. Their invariance is violated near

$$
\zeta \approx C\left(J_{\max }-2 \hat{\mu}\right)^{1 / 2}
$$

at which point $J_{\rho}$ changes by a factor of $\sim 2$. (See Figure 12). This neglects a possible interaction between the $\rho$ and $\zeta$ degrees-of-freedom. The values of $\zeta$ for which $\mu$ is violated are shown in Figure 7. For figure- 8 orbits, it sets the limit on the highest possible amplitude of oscillation in one of the two symmetric potential wells that prescribe averaged motion along the $\zeta$-axis.

The stochasticity criterion given by Eq. (35) can be used to calculate the percentage of stochastic particles for a given distribution of energies and angular momenta. For example, a low energy beam parallel to the $\zeta$ axis with $V_{0} \pi_{\zeta}^{2} \ll T$, where $T$ is the perpendicular temperature, with $T=V_{0} v\left(\rho_{h}, 0, \pi_{\phi}\right)$ and $\pi_{\phi}=1 / 5$ will have 85 percent regular orbits (regular meaning those that adiabatically conserve $J_{\rho}$. This calculation was performed by finding the percentage of figure- 8 orbits for this distribution. This particular calculation can be used when the parallel energy of the beam can be assumed to be essentially zero, relative to perpedicular temperature. In this case, since $\pi_{\zeta} \approx 0$, all figure- 8 orbits of the 
initial distribution are either at the critical amplitude, or at the turning point of oscillation about $\zeta=0$.

Figure 13 shows action-angle $\left(J_{\rho}\right.$ and $\left.\theta_{\rho}\right)$ Poincaré plots on the $\zeta=0$ section for a range of $J_{\rho}$ values. The selection of the $\zeta=0$ plane eliminates inclusion of particles trapped in the symmetric-in- $\zeta$ potential wells, e.g. Figures 8a), 10), and 11). Each color represents a different initial condition (different $J_{\rho}$ values at fixed $\pi_{\phi}$ ). Taking the $J_{\rho}$ and $\theta_{\rho}$ variables as being on the smaller cross-section of a torus, we can see that for values of (the scaled) $J_{\rho}$ less that 0.095, the dots representing cyclotron orbits show stochastic behavior. At higher values of $J_{\rho}$, closed circles occur, representing figure- 8 orbits, showing that the motion is integrable. The set of clearly-defined closed circles $c-g$, representing integrable motion, begins just above the critical value of $J_{\rho}$, i.e., circle $b$ Figure 13, and are part of a continuous set of closed KAM curves. $^{22}$ The circle labeled $b$ corresponds to figure- 8 orbits with just enough energy to cross the central barrier of $V_{\text {eff }}$. The integrability of orbits which execute a figure- 8 orbit at $\zeta=0$ is expressed in the condition given by Eq. (29). Since these orbits never undergo a transition, there is no limit on their amplitude of oscillation along $\zeta$.

A gap in the action-angle Poincaré plot in Figure 13 separates stochastic and integrable motion. It is due to the fact that the lowest energy figure- 8 orbit has $J_{\rho}$ about twice as big as that of the highest energy cyclotron orbit, because the figure- 8 orbit oscillates across two potential wells and cyclotron orbit across one. Thus the radius of the $b$ circle in Figure 13 is about twice as big as that of the $a$ circle.

\section{CONCLUSIONS}

The dynamics of single ions in a large aspect ratio FRC were explored. A fuller description of ion orbits in the $z=\zeta=0$ subspace was presented. Stability of these orbits to out-ofplane perturbations was shown to depend on $\left\langle\rho^{2} \dot{\phi}\right\rangle$. For integrable orbits, $\omega_{\rho} \gg \omega_{\zeta}$, the averaged motion is in a single or double symmetric potential along the major axis, $\zeta$. The locations of the minima along $\zeta$ of this averaged potential are a function of the invariants $J_{\rho}$ and conserved $\pi_{\phi}$. Higher values of $J_{\rho}$ and $\pi_{\phi}$ lead to closer spaced double potential wells, until the two wells merge into a single potential centered around $\zeta=0$. To be integrable, an orbit must either have a high enough energy along $\rho$ to execute figure- 8 or betatron motion in the $\zeta=0$ cross-section or be trapped in one of two averaged potentials along $\zeta$. Two 
criteria for stochastic trajectories were derived. The first criterion, Eq. (29), for untrapped orbits, was shown to depend on $J_{\rho}$ and $\pi_{\phi}$. The second criterion is given by Eq. (35), and ensures that the amplitude of oscillation along $\zeta$ in one of two potential wells is low enough so that the ion doesn't experience a transition to cyclotron motion. It is found that the amplitude of integrable oscillations along $\zeta$ decreases with decreasing $J_{\rho}$, and goes to zero as the cyclotron radius goes to zero. Since $J_{\rho}$ determines the shape of averaged potential along $\zeta$, for a given value of $\pi_{\phi}$, particle dynamics can be represented on a two-dimensional torus with Poincaré cross-section of $\theta_{\zeta}$ and $J_{\zeta}$. For low values of $J_{\zeta}$ (amplitudes of oscillation along $\zeta$ below the critical value given by Eqs. (35)), $J_{\zeta}$ is conserved and Poincaré crosssections show closed circles. The onset of stochastic behavior which occurs at higher values of $J_{\zeta}$ and island formation can be studied using KAM and perturbation theories, where the Hamiltonian is expanded around minima in $\zeta$ estimated by Eq. (23). Different values of $J_{\rho}$ give a family of nested tori.

Acknowledgements: This work was supported, in part, by U.S. Department of Energy Contract No. DE-AC02-76-CHO-3073. 


\section{References}

* Electronic address: <landsman@princeton.edu>

1 M. S. Chance, J. M. Greene, and T. H. Jensen, Geophys. Fluid Dynamics 65, 203 (1992).

2 H. Momota, A. Ishida, and Y. Kohzaki, Fusion Technology 21, 701 (1992).

3 R. Lovelace, D. Larrabee, and H. Fleischmann, Phys. Fluids 23, 1436 (1980).

4 M. Tuszewski, Nucl. Fusion 28, 28 (1988).

5 M. Tuszewski, D. P. Taggart, R. Chrien, D. J. Rej, R. E. Siemon, and B. L. Wright, Phys. Fluids B3, 2856 (1991).

6 W. Armstrong, R. Linford, J.Lipson, D. A. Platts, and E. G. Sherwood, Phys. Fluids 24, 2068 (1981).

7 J. Slough and K. Miller, Phys. Rev. Lett. 85, 1444 (2000).

8 M. N. Rosenbluth and M. N. Bussac, Nucl. Fusion 19, 489 (1979).

9 M. Y. Wang and G. H. Miley, Nucl. Fusion 19, 39 (1979).

10 J. M. Finn, Phys. Fluids 24, 274 (1980).

11 J. M. Finn and R. N. Sudan, Nucl. Fusion 22, 1443 (1982).

12 J.-S. Kim and J. R. Cary, Phys. Fluids (1983).

13 E.V. Belova, R.C. Davidson H. Ji and M. Yamada, Phys. Plasmas (2003).

14 H. Yumi, T. Toshiki, and K. Yoshiomi, Nucl. Fusion 42, 1075 (2002).

15 W. Hugrass and M. Turley, J. Plasma Phys. 37, 1 (1987).

16 S. A. Cohen and A. H. Glasser, Phys. Rev. Lett. 85, 5114 (2000).

17 A. H. Glasser and S. A. Cohen, Phys. Plasmas 9, 2093 (2002).

18 A. I. Neishtadt, Sov. J. Plasma Phys. 12, 568 (1986).

19 J. R. Cary, D. F. Escande, and J. L. Tennyson, Phys. Rev. A 34, 4256 (1986).

20 L. S. Solov'ev, Rev. Plasma Phys. 6, 239 (1976).

21 G. M. Zaslavsky, Physics of chaos in Hamiltonian systems (Imperial College Press, London, 1998).

22 A. J. Lichtenberg and M. A. Lieberman, Regular and Chaotic Dynamics (Springer-Verlag, New York, 1992). 


\section{List of Figures}

Figure 1: Possible shapes of the scaled effective potential

Figure 2: Particle orbits

Figure 3: Cross-sections of potential for different values of $\zeta$

Figure 4: Phase-space plots showing single or double averaged potential along $\zeta$

Figure 5: Comparison of $\rho_{h}^{2}\left(1-\rho_{h}^{2}\right)$ and $\pi_{\phi}$

Figure 6: Comparison of analysis and numerical simulation for averaged kinetic energy along $\zeta$

Figure 7: Boundary for the crossing of the separatrix

Figure 8: Regular motion for trapped particle orbits

Figure 9: Stochastic motion for trapped particle orbits

Figure 10: Oscillation at the critical amplitude at higher value of $J_{\rho}$

Figure 11: Oscillation at the critical amplitude at lower value of $J_{\rho}$

Figure 12: Change in $J_{\rho}$ during a transition between cyclotron and figure-8 orbits

Figure 13: Poincaré plot in Action-Angle variables 


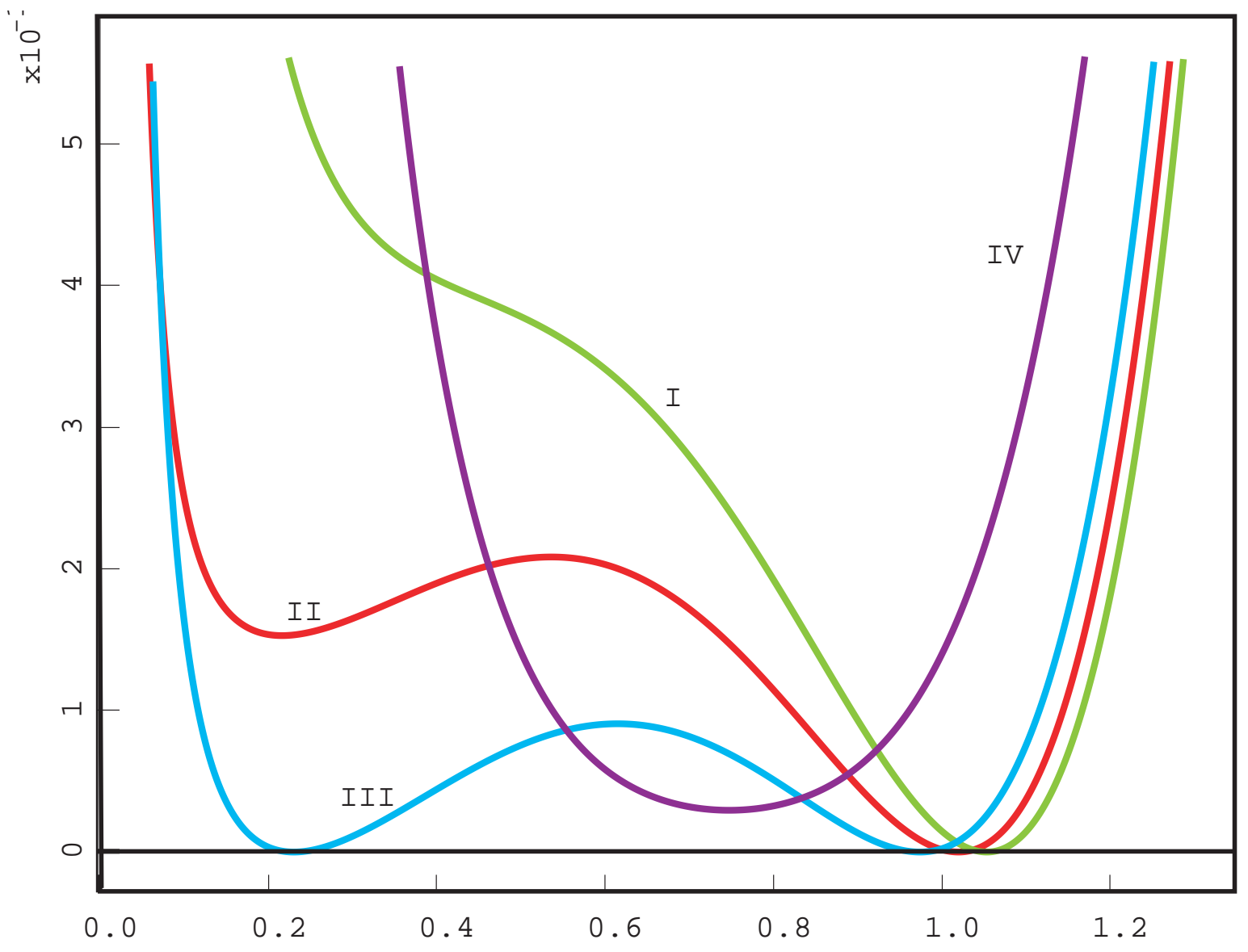

FIG. 1: Possible shapes of the scaled effective potential $v\left(\rho, 0, \pi_{\phi}\right)$ as a function of the scaled radius $\rho$ in the $\zeta=\pi_{\zeta}=0$ invariant subspace for four values the scaled angular momentum $\pi_{\phi}$, representative of four distinct cases. I: $\pi_{\phi}=-0.12$, single asymmetric well touching zero; II: $\pi_{\phi}=-0.04$, asymmetric double well; III: $\pi_{\phi}=0.05$, double potential well with both minima touching zero; IV: $\pi_{\phi}=0.375$, raised potential well. 

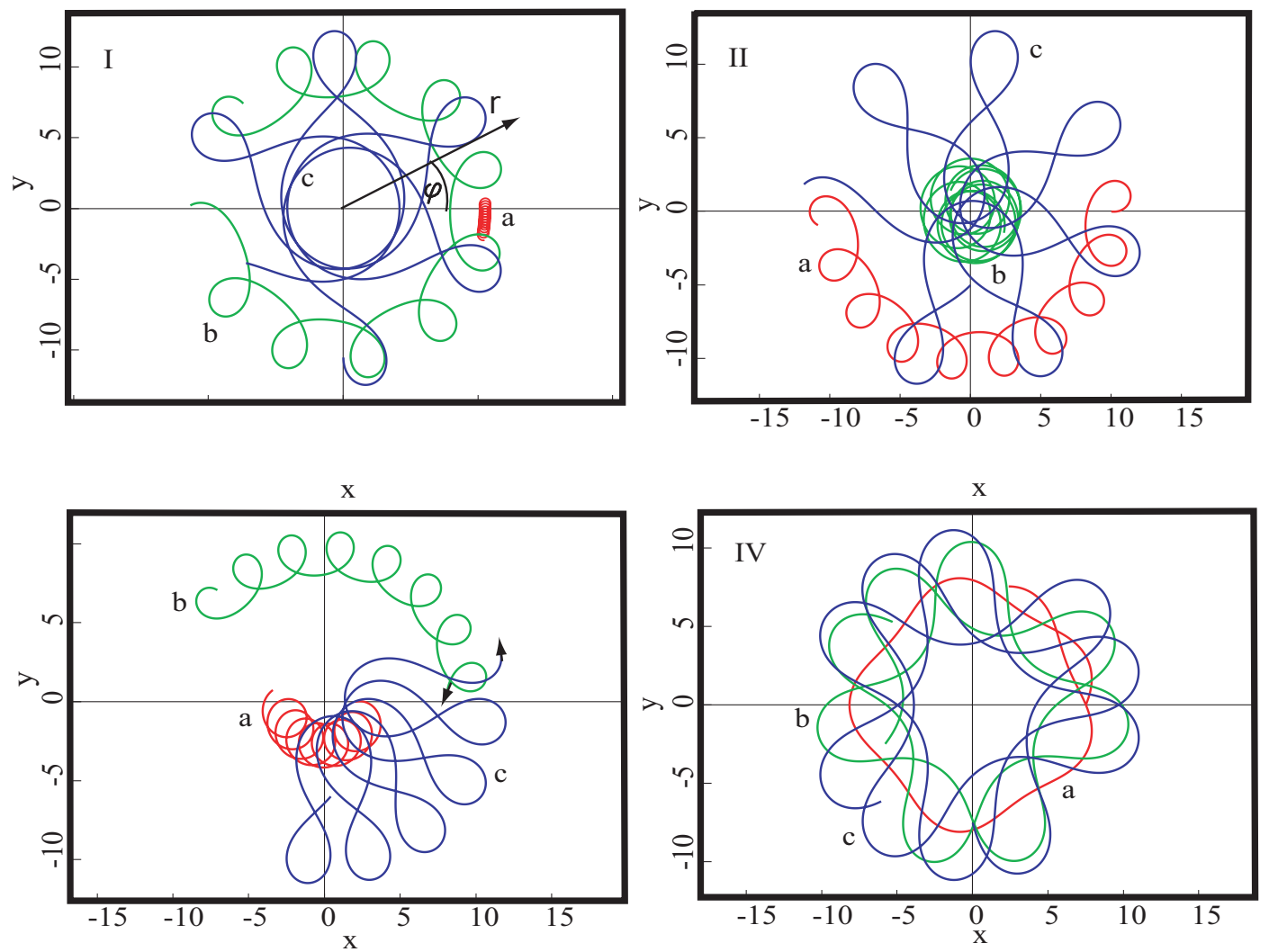

FIG. 2: Particle orbits in the potentials of Figure 1 with $r_{s}=10 \mathrm{~cm}$. I: scaled energy $\varepsilon=0.01(a), 0.2(b), 0.4(c) ;$ II: $\varepsilon=0.1(a), 0.18(b), 0.4(c) ;$ III: $\varepsilon=.05(a), 0.05(b), 0.2(c) ;$ IV: $\varepsilon=0.035(a), 0.2(b), 0.4(c)$. 


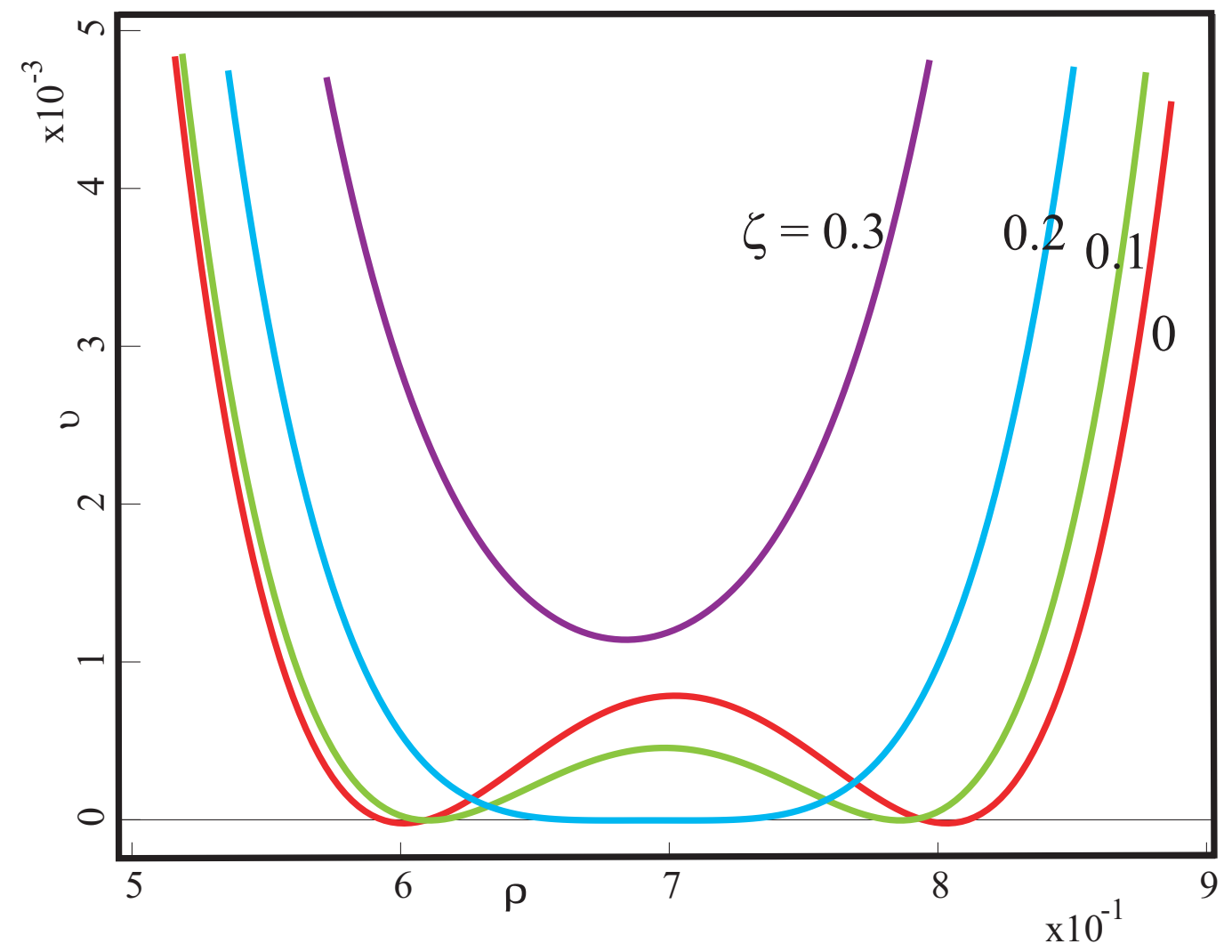

FIG. 3: $v\left(\rho, \zeta, \pi_{\phi}\right)$ vs. $\rho$ for $\pi_{\phi}=0.23$, Case III, with $\zeta=0.0,0.1,0.2$, and 0.3. For $\pi_{\phi}>0$, the barrier in the double potential drops as $|\zeta|$ increases, until $v\left(\rho, \zeta, \pi_{\phi}\right)$ turns into a single raised potential well. $\dot{\phi}$ is negative in the interval between the two zeroes and positive elsewhere. For the raised potential $\dot{\phi}$ is everywhere positive. 

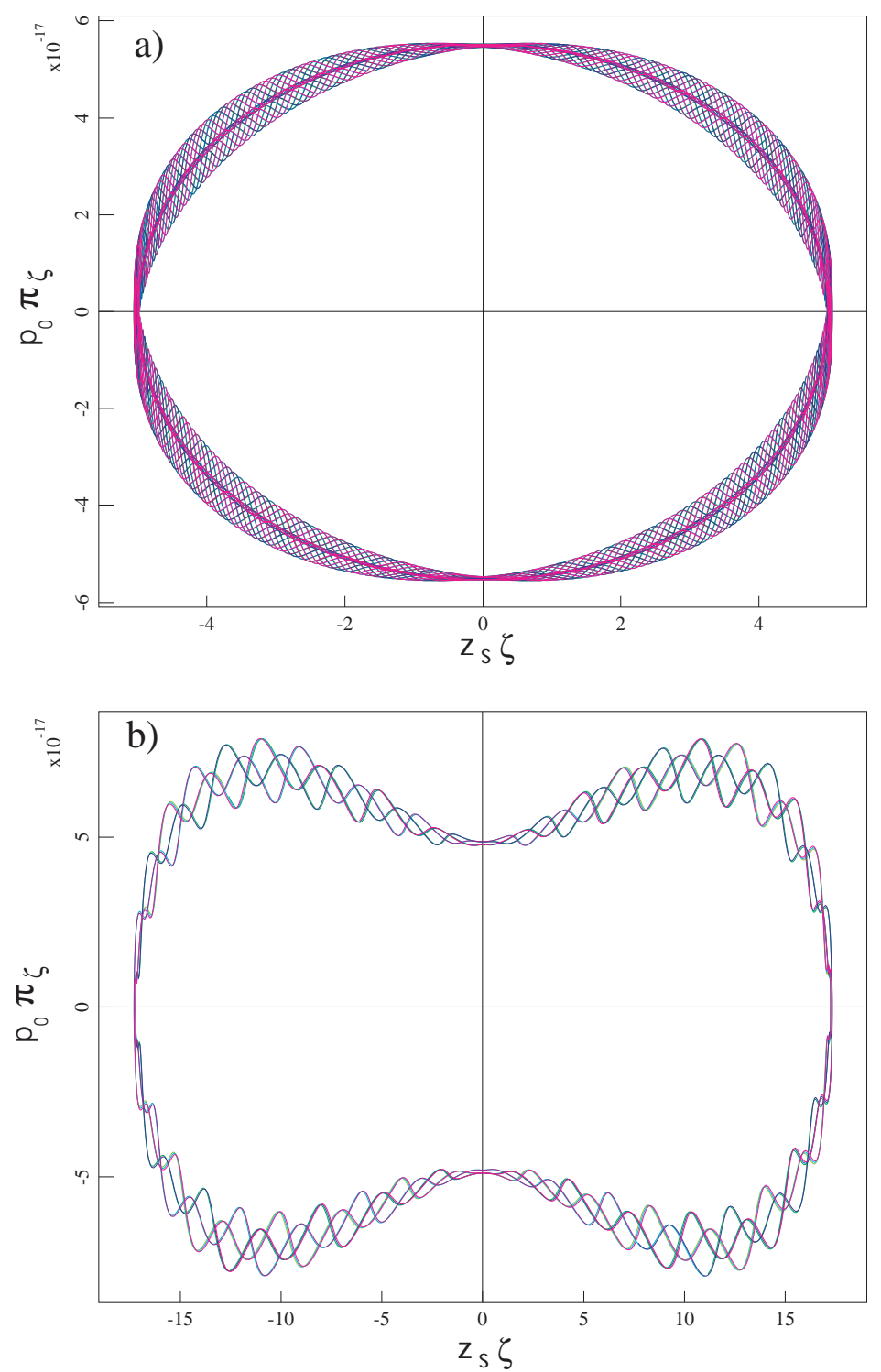

FIG. 4: Phase-space plots, $\pi_{\zeta}$ vs. $\zeta$, for a high and a low radial-energy ion having regular motion. a) High radial energy: The shape of averaged potential along $\zeta$ is a single potential well. b) Low radial energy: The shape of averaged potential along $\zeta$ is a double potential well. 


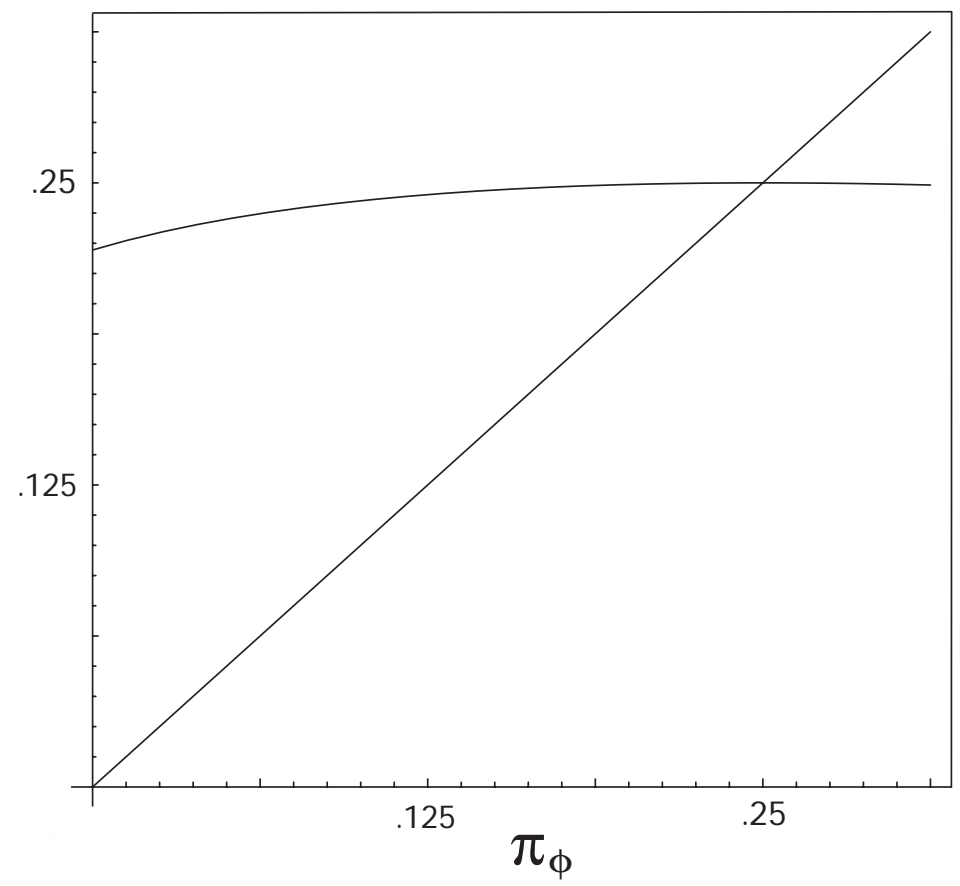

FIG. 5: $\rho_{h}^{2}\left(1-\rho_{h}^{2}\right)$ and $\pi_{\phi}$, vs $\pi_{\phi}$. Intersection occurs at the critical point, $\pi_{\phi}=1 / 4$. 


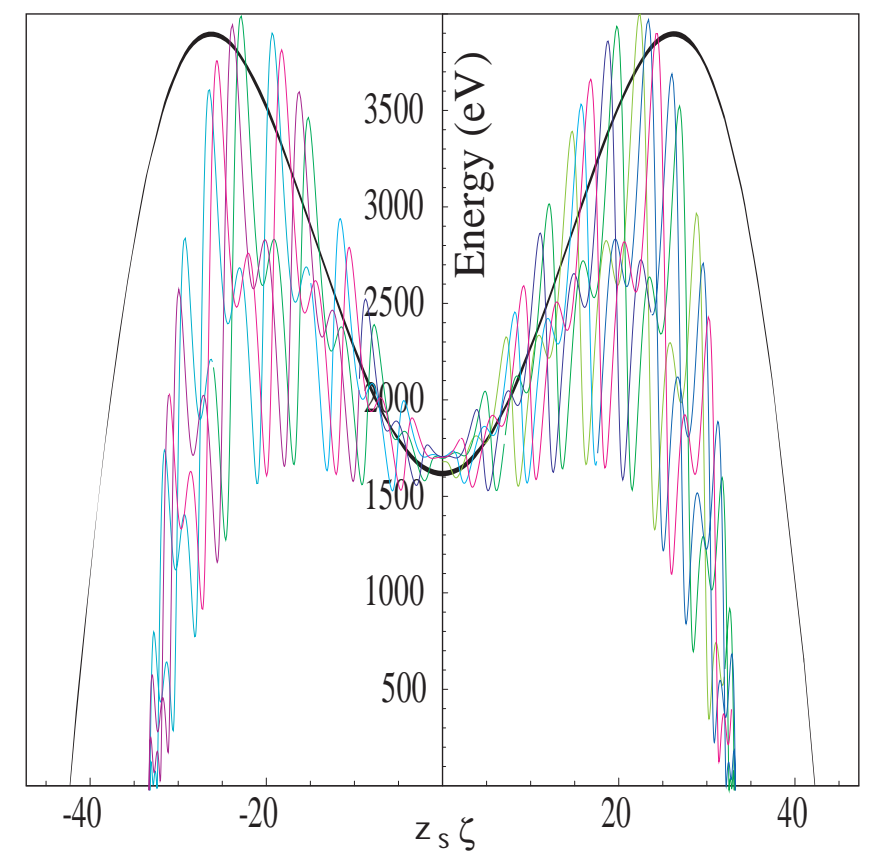

FIG. 6: The solid black line is the averaged $\zeta$-directed kinetic energy, $V_{0}<\pi_{\zeta}^{2}>$, obtained using Eq. (17) with $\rho_{h}$ given by Eq. (21). The oscillating curve is $V_{0} \pi_{\zeta}^{2}$ obtained from the full-Hamiltonian numerical simulation. In both cases $\pi_{\phi}=1 / 16, \epsilon=0.2$, and amplitude of oscillation along $\rho$ is $A=.5028$. The full-Hamiltonian simulation shows fast $\rho$ oscillations superimposed on the averaged potential. 


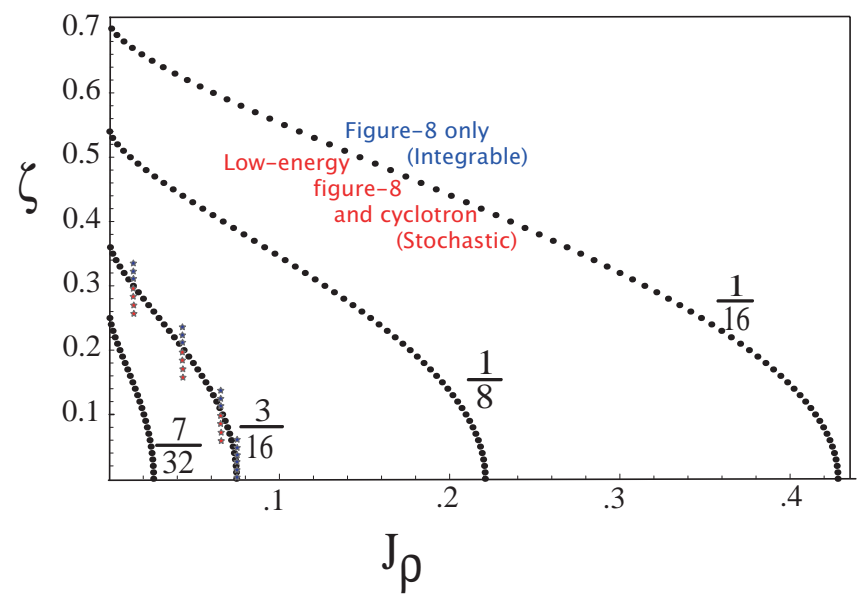

FIG. 7: The curves on the graph indicate the location along $\zeta$ where the separatrix is approached for corresponding values of $J_{\rho}$. Each curve corresponds to a different value of $\pi_{\phi}$, where $\pi_{\phi}=$ $1 / 16,1 / 8,3 / 16,7 / 32$. Lower values of $\pi_{\phi}$ have higher $\zeta$ intercepts. The curve disappears at the critical value $\pi_{\phi}=\frac{1}{4}$. For $\pi_{\phi}=3 / 16$, numerical simulations of orbits were made at four values of $J_{\rho}$ at closely spaced values of $\zeta$. The red stars indicate cases where the orbits were stochastic and the blue stars where the orbits were integrable. For $\pi_{\phi}=1 / 16$, the region above the curve is marked as containing regular (figure-8) orbits and that below containing stochastic (both figure-8 and cyclotron) orbits. 

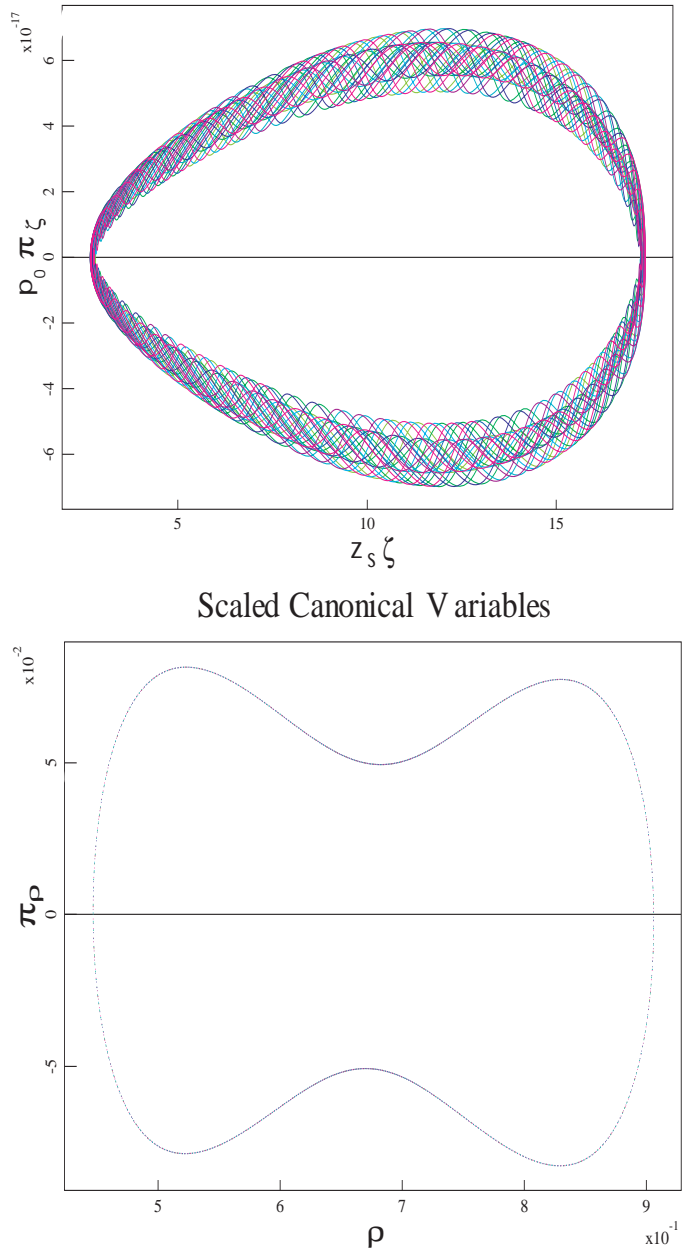

FIG. 8: Regular motion: Oscillation in one of the two minima in $\zeta,(\epsilon=1 / 5)$ with amplitude of $\zeta$ motion just below the critical amplitude. The lower figure is a Poincaré surface-of-section. 

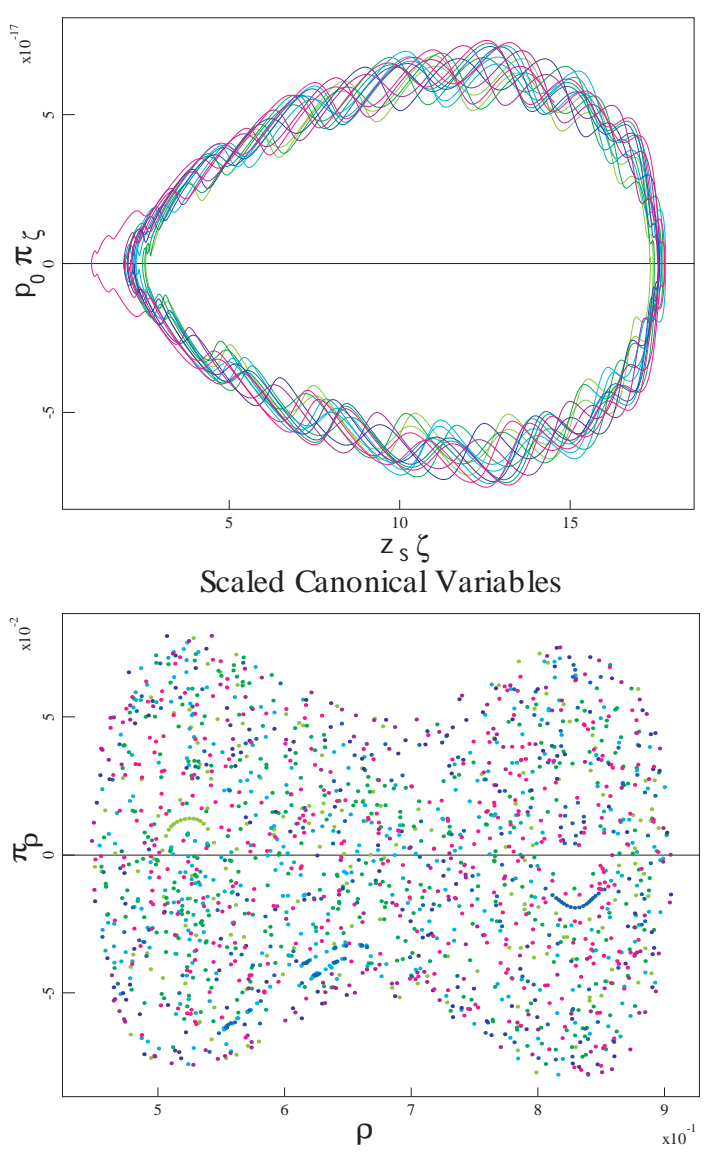

FIG. 9: Stochastic motion: The amplitude of oscillation in one of the two minima along $\zeta(\epsilon=1 / 5)$ was above the critical, resulting in chaotic motion. The lower figure is a Poincaré surface-of-section. 

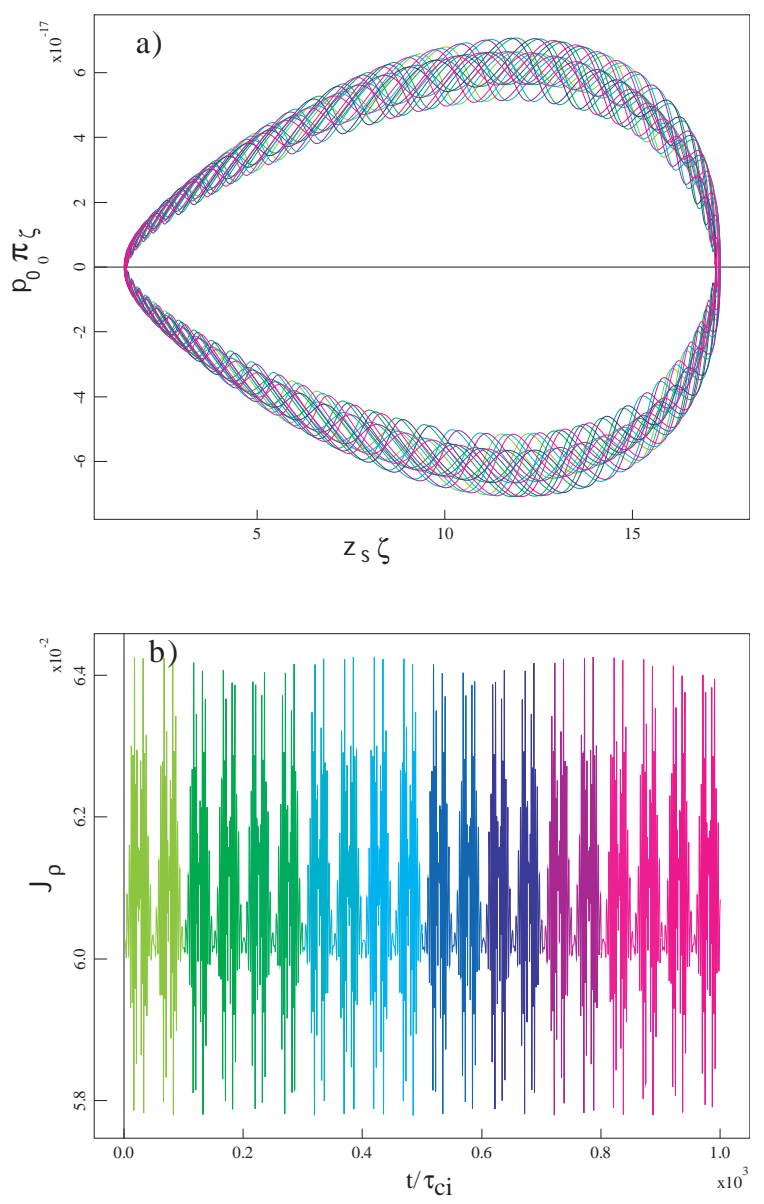

FIG. 10: a) Phase-space plot for oscillation at the critical amplitude for $J_{\rho} \approx 0.061$. b) $J_{\rho} v s$. $\tau_{c i}$ in units of $\tau_{c i}$, the ion cyclotron period at $\zeta=\rho=0$. Since the orbit is integrable, $J_{\rho}$ is approximately conserved. 

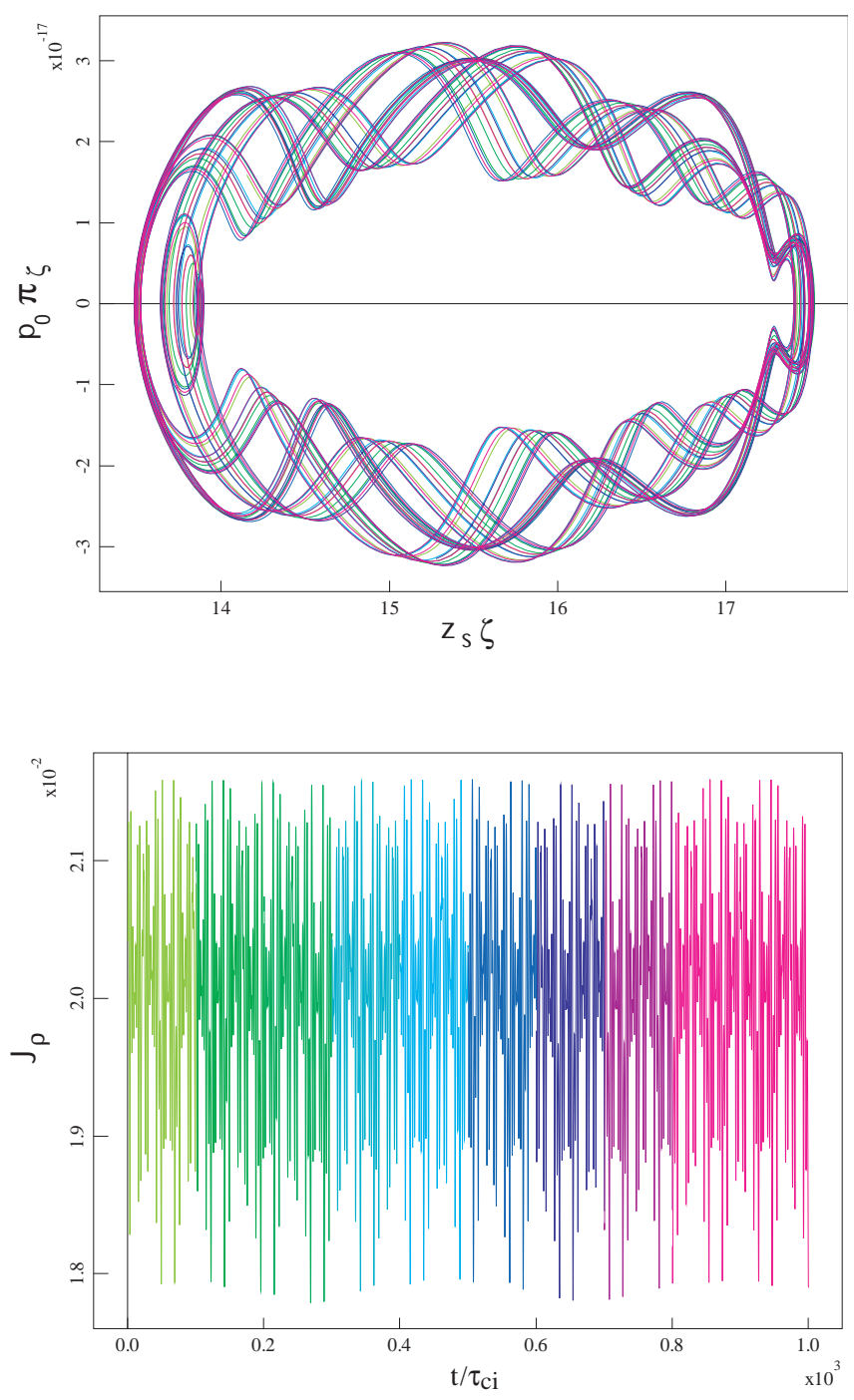

FIG. 11: a) Phase-space plot for oscillation at the critical amplitude for $J_{\rho} \approx 0.02$. The critical amplitude of oscillation decreases with decreasing value of $J_{\rho}$ (compare with Figure 10) b) $J_{\rho} v s$. $t / \tau_{c i}$. Since the orbit is integrable, $J_{\rho}$ is approximately conserved. 


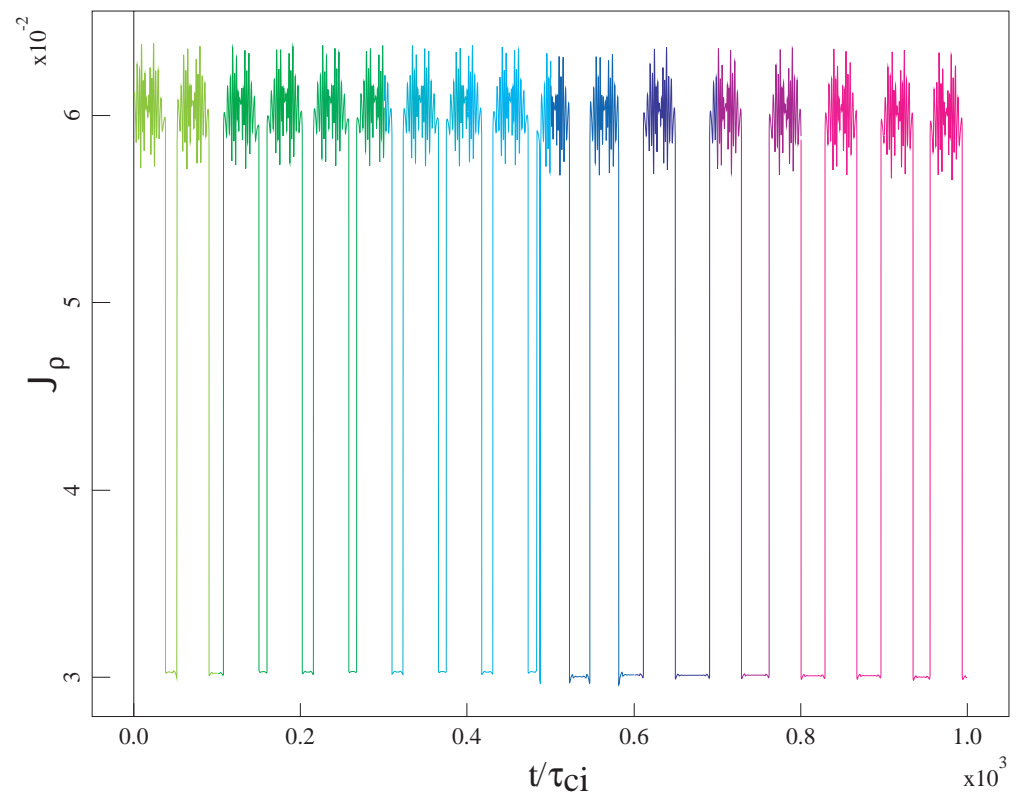

FIG. 12: $J_{\rho}$ goes up by approximately a factor of 2 (neglecting interaction between $\rho$ and $\zeta$ degrees of freedom) during a transition of a cyclotron to a figure- 8 orbit. 


\section{Scaled Action-Angle V ariables}

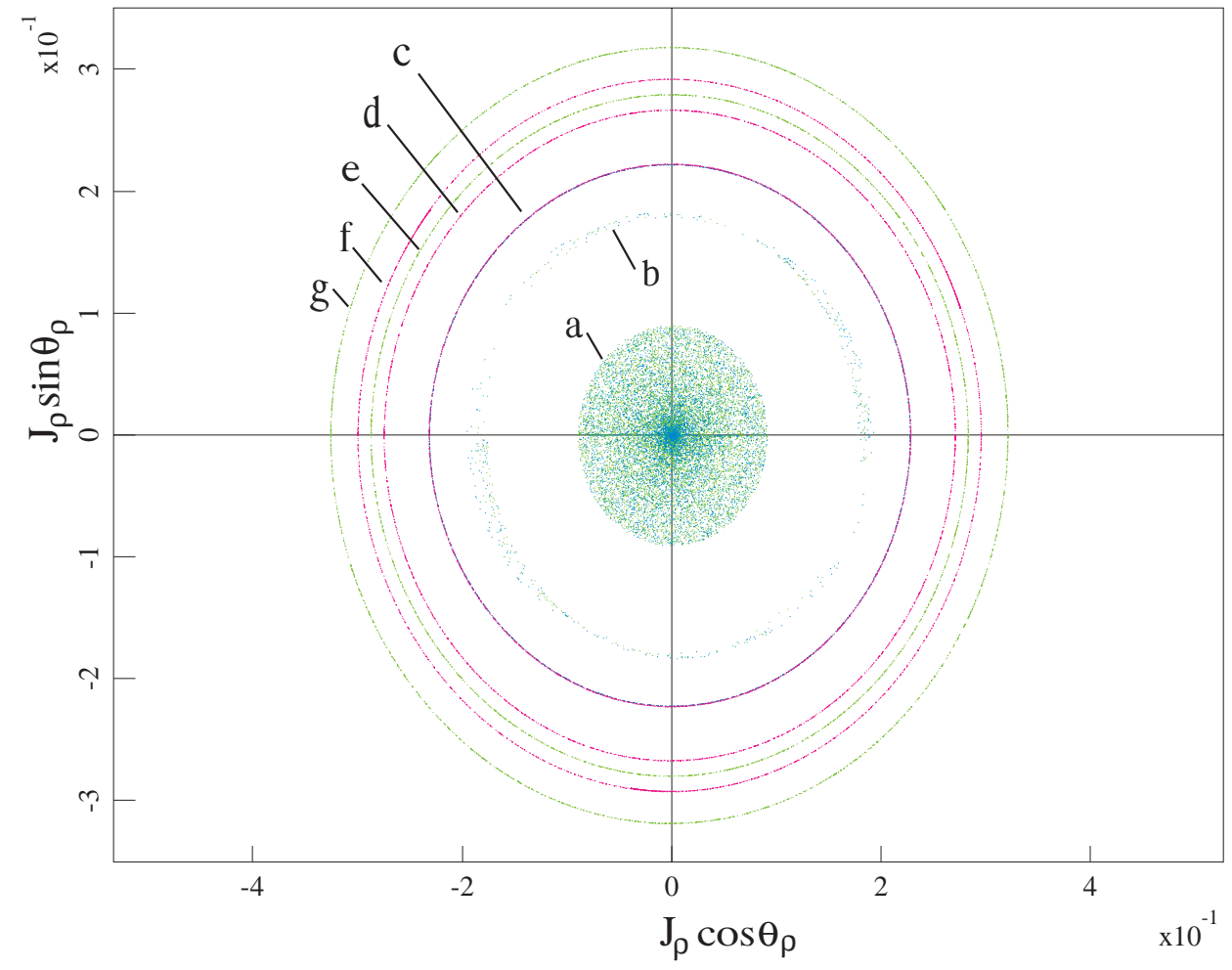

FIG. 13: Poincaré plot in Action-Angle variables taken in $\zeta=0$ cross-section: $x=J_{\rho} \cos \theta_{\rho}$, $y=J_{\rho} \sin \theta_{\rho}$. 


\section{External Distribution}

Plasma Research Laboratory, Australian National University, Australia

Professor I.R. Jones, Flinders University, Australia

Professor João Canalle, Instituto de Fisica DEQ/IF - UERJ, Brazil

Mr. Gerson O. Ludwig, Instituto Nacional de Pesquisas, Brazil

Dr. P.H. Sakanaka, Instituto Fisica, Brazil

The Librarian, Culham Laboratory, England

Mrs. S.A. Hutchinson, JET Library, England

Professor M.N. Bussac, Ecole Polytechnique, France

Librarian, Max-Planck-Institut für Plasmaphysik, Germany

Jolan Moldvai, Reports Library, Hungarian Academy of Sciences, Central Research Institute for Physics, Hungary

Dr. P. Kaw, Institute for Plasma Research, India

Ms. P.J. Pathak, Librarian, Institute for Plasma Research, India

Ms. Clelia De Palo, Associazione EURATOM-ENEA, Italy

Dr. G. Grosso, Instituto di Fisica del Plasma, Italy

Librarian, Naka Fusion Research Establishment, JAERI, Japan

Library, Laboratory for Complex Energy Processes, Institute for Advanced Study, Kyoto University, Japan

Research Information Center, National Institute for Fusion Science, Japan

Dr. O. Mitarai, Kyushu Tokai University, Japan

Dr. Jiangang Li, Institute of Plasma Physics, Chinese Academy of Sciences, People's Republic of China

Professor Yuping Huo, School of Physical Science and Technology, People's Republic of China

Library, Academia Sinica, Institute of Plasma Physics, People's Republic of China

Librarian, Institute of Physics, Chinese Academy of Sciences, People's Republic of China

Dr. S. Mirnov, TRINITI, Troitsk, Russian Federation, Russia

Dr. V.S. Strelkov, Kurchatov Institute, Russian Federation, Russia

Professor Peter Lukac, Katedra Fyziky Plazmy MFF UK, Mlynska dolina F-2, Komenskeho Univerzita, SK-842 15 Bratislava, Slovakia

Dr. G.S. Lee, Korea Basic Science Institute, South Korea

Institute for Plasma Research, University of Maryland, USA

Librarian, Fusion Energy Division, Oak Ridge National Laboratory, USA

Librarian, Institute of Fusion Studies, University of Texas, USA

Librarian, Magnetic Fusion Program, Lawrence Livermore National Laboratory, USA

Library, General Atomics, USA

Plasma Physics Group, Fusion Energy Research Program, University of California at San Diego, USA

Plasma Physics Library, Columbia University, USA

Alkesh Punjabi, Center for Fusion Research and Training, Hampton University, USA

Dr. W.M. Stacey, Fusion Research Center, Georgia Institute of Technology, USA

Dr. John Willis, U.S. Department of Energy, Office of Fusion Energy Sciences, USA

Mr. Paul H. Wright, Indianapolis, Indiana, USA 
The Princeton Plasma Physics Laboratory is operated by Princeton University under contract with the U.S. Department of Energy.

\author{
Information Services \\ Princeton Plasma Physics Laboratory \\ P.O. Box 451 \\ Princeton, NJ 08543
}

Phone: 609-243-2750

Fax: 609-243-2751

e-mail: pppl_info@pppl.gov

Internet Address: http://www.pppl.gov 